\title{
Deformation and mixing of coexisting shapes in neutron-deficient polonium isotopes
}

N. Kesteloot, ${ }^{1,2,{ }^{*}}$ B. Bastin,,${ }^{1,3}$ L. P. Gaffney, ${ }^{1,4}$ K. Wrzosek-Lipska, ${ }^{1,5}$ K. Auranen,,${ }^{6,7}$ C. Bauer, ${ }^{8}$ M. Bender, ${ }^{9,10}$ V. Bildstein, ${ }^{11}$ A. Blazhev, ${ }^{12}$ S. Bönig, ${ }^{8}$ N. Bree,${ }^{1}$ E. Clément, ${ }^{3}$ T. E. Cocolios, ${ }^{1,13,14}$ A. Damyanova, ${ }^{15}$ I. Darby, ${ }^{1,6,7}$ H. De Witte, ${ }^{1}$ D. Di Julio, ${ }^{16}$ J. Diriken, ${ }^{1,2}$ C. Fransen, ${ }^{12}$ J. E. García-Ramos, ${ }^{17}$ R. Gernhäuser, ${ }^{11}$ T. Grahn, ${ }^{6,7}$ P.-H. Heenen, ${ }^{18}$ H. Hess, ${ }^{12}$ K. Heyde, ${ }^{19}$ M. Huyse, ${ }^{1}$ J. Iwanicki, ${ }^{5}$ U. Jakobsson, ${ }^{6,7}$ J. Konki, ${ }^{6,7}$ T. Kröll, ${ }^{8}$ B. Laurent, ${ }^{20}$ N. Lecesne, ${ }^{3}$ R. Lutter, ${ }^{21}$ J. Pakarinen, ${ }^{6,7}$ P. Peura, ${ }^{6,7}$ E. Piselli, ${ }^{13}$ L. Próchniak, ${ }^{5}$ P. Rahkila, ${ }^{6,7}$ E. Rapisarda, ${ }^{1,13}$ P. Reiter, ${ }^{12}$ M. Scheck, ${ }^{4,8,22,23}$ M. Seidlitz, ${ }^{12}$ M. Sferrazza, ${ }^{24}$ B. Siebeck, ${ }^{12}$ M. Sjodin, ${ }^{3}$ H. Tornqvist, ${ }^{13}$ E. Traykov, ${ }^{3}$ J. Van De Walle, ${ }^{13}$ P. Van Duppen, ${ }^{1}$ M. Vermeulen, ${ }^{25}$ D. Voulot, ${ }^{13}$ N. Warr, ${ }^{12}$ F. Wenander, ${ }^{13}$ K. Wimmer, ${ }^{11}$ and M. Zielińska ${ }^{5,20}$

${ }^{1}$ Instituut voor Kern- en Stralingsfysica, KU Leuven, B-3001 Leuven, Belgium

${ }^{2}$ Belgian Nuclear Research Centre SCK $\bullet$ CEN, B-2400 Mol, Belgium

${ }^{3}$ GANIL CEA/DSM-CNRS/IN2P3, Boulevard H. Becquerel, F-14076 Caen, France

${ }^{4}$ Oliver Lodge Laboratory, University of Liverpool, Liverpool L69 7ZE, United Kingdom

${ }^{5}$ Heavy Ion Laboratory, University of Warsaw, PL-02-093 Warsaw, Poland

${ }^{6}$ Department of Physics, University of Jyväskylä, P.O. Box 35, FI-40014 Jyväskylä, Finland

${ }^{7}$ Helsinki Institute of Physics, P.O. Box 64, FI-00014 Helsinki, Finland

${ }^{8}$ Institut für Kernphysik, Technische Universität Darmstadt, D-64289 Darmstadt, Germany

${ }^{9}$ Université Bordeaux, Centre d'Etudes Nucléaires de Bordeaux Gradignan, UMR5797, F-33175 Gradignan, France

${ }^{10}$ CNRS/IN2P3, Centre d'Etudes Nucléaires de Bordeaux Gradignan, UMR5797, F-33175 Gradignan, France

${ }^{11}$ Physics Department E12, Technische Universität München, D-85748 Garching, Germany

${ }^{12}$ Institut für Kernphysik, Universität zu Köln, 50937 Köln, Germany

${ }^{13}$ ISOLDE, CERN, CH-1211 Geneva 23, Switzerland

${ }^{14}$ School of Physics \& Astronomy, The University of Manchester, Manchester M13 9PL, United Kingdom

${ }^{15}$ Université de Genève, 24 Quai Ernest-Ansermet, $\mathrm{CH}-1211$ Genève 4, Switzerland

${ }^{16}$ Physics Department, University of Lund, Box 118, SE-22100 Lund, Sweden

${ }^{17}$ Departamento de Física Aplicada, Universidad de Huelva, 21071 Huelva, Spain

${ }^{18}$ Physique Nucléaire Théorique, Université Libre de Bruxelles, B-1050 Bruxelles, Belgium

${ }^{19}$ Department of Physics and Astronomy, Ghent University, Proeftuinstraat 86, B-9000 Ghent, Belgium

${ }^{20}$ IRFU/SPhN, CEA Saclay, F-91191 Gif-sur-Yvette, France

${ }^{21}$ Ludwig-Maximilians-Universität-München, Schellingstraße 4, 80799 München, Germany

${ }^{22}$ School of Engineering, University of the West of Scotland, Paisley PA1 2BE, United Kingdom

${ }^{23}$ SUPA, Scottish Universities Physics Alliance, Glasgow G12 8QQ, United Kingdom

${ }^{24}$ Département de Physique, Faculté des Sciences, Université Libre de Bruxelles (ULB), Boulevard du Triomphe, 1050 Brussels, Belgium

${ }^{25}$ Department of Physics, The University of York, York YO10 5DD, United Kingdom

(Received 19 January 2015; published 4 November 2015)

\begin{abstract}
Coulomb-excitation experiments are performed with postaccelerated beams of neutron-deficient ${ }^{196,198,200,202} \mathrm{Po}$ isotopes at the REX-ISOLDE facility. A set of matrix elements, coupling the low-lying states in these isotopes, is extracted. In the two heaviest isotopes, ${ }^{200,202} \mathrm{Po}$, the transitional and diagonal matrix elements of the $2_{1}^{+}$state are determined. In ${ }^{196,198}$ Po multistep Coulomb excitation is observed, populating the $4_{1}^{+}, 0_{2}^{+}$, and $2_{2}^{+}$states. The experimental results are compared to the results from the measurement of mean-square charge radii in polonium isotopes, confirming the onset of deformation from ${ }^{196} \mathrm{Po}$ onwards. Three model descriptions are used to compare to the data. Calculations with the beyond-mean-field model, the interacting boson model, and the general Bohr Hamiltonian model show partial agreement with the experimental data. Finally, calculations with a phenomenological two-level mixing model hint at the mixing of a spherical structure with a weakly deformed rotational structure.
\end{abstract}

DOI: 10.1103/PhysRevC.92.054301

PACS number(s): 25.70.De, 23.20.Js, 25.60.-t, 27.80.+w

\section{INTRODUCTION}

Nuclear shape coexistence is the remarkable phenomenon in which states at similar excitation energies exhibit different

\footnotetext{
*nele.kesteloot@fys.kuleuven.be
}

Published by the American Physical Society under the terms of the Creative Commons Attribution 3.0 License. Further distribution of this work must maintain attribution to the author(s) and the published article's title, journal citation, and DOI. intrinsic deformations. By now it is established to appear throughout the whole nuclear landscape, in light, medium, and heavy nuclei [1]. A substantial number of data have been gathered in the neutron-deficient lead region, providing clear evidence for the coexistence of shapes in these nuclei from an experimental as well as a theoretical point of view. 
Experimentally, shape coexistence is well established in mercury isotopes $(Z=80)$ around neutron midshell, e.g., the large odd-even staggering and large isomer shift in the measured charge radii [2]. Despite the relatively constant behavior of the $2_{1}^{+}$energy and of the reduced transition probabilities $B\left(E 2 ; 2_{1}^{+} \rightarrow 0_{1}^{+}\right)$, a recent Coulomb-excitation study of the neutron-deficient, even-even ${ }^{182-188} \mathrm{Hg}$ isotopes led to the interpretation of mixing between two different structures that coexist at a low excitation energy [3]. Mixing between a weakly deformed oblate-like band and a more deformed prolate-like band is proposed to gain importance when going towards neutron midshell nuclei. This mixing between two configurations is also predicted in recent theoretical efforts studying neutron-deficient mercury isotopes in the framework of the interacting boson model (IBM) with configuration mixing [4].

The ${ }^{186} \mathrm{~Pb}$ nucleus $(Z=82)$ is a unique case of shape coexistence since three $0^{+}$states with different deformations have been observed within an energy span of $700 \mathrm{keV}$ [5]. Also, many other lead isotopes display signs of shape coexistence [6]. However, the ground states of the neutron-deficient lead isotopes are found to stay essentially spherical while different shapes appear at low excitation energies $[7,8]$.

In the polonium isotopes, above $Z=82$, low-lying intruder states have also been identified. Early theoretical studies concluded that the ground state of the heavier ${ }^{194-210} \mathrm{Po}$ isotopes remains spherical, with the first (oblate-like) deformed ground state appearing in ${ }^{192} \mathrm{Po}$ [9]. A prolate deformation in the ground state was suggested for the lightest polonium isotopes with mass $A \leqslant 190$. These findings were supported by a series of experimental studies of the polonium isotopes employing a range of techniques that include $\alpha-, \beta$-, and in-beam $\gamma$-decay studies (e.g., see Refs. [6] and [10]). The intrusion of the deformed state, becoming the ground state, is an unexpected result as in the even-even mercury isotopes, which "mirror" the polonium isotopes with respect to $Z=82$, the intruding $0^{+}$deformed state never becomes the groundstate structure.

Recent results from the measurement of changes in meansquare charge radii $\delta\left\langle r^{2}\right\rangle$ in a wide range of polonium isotopes point to an onset of deviation from sphericity around ${ }^{198} \mathrm{Po}$ [8,11], which is significantly earlier, when going towards a lighter mass, than previously suggested (e.g., in [6]). Comparison of the mean-square charge radii of the polonium isotopes with their isotones below $Z=82$, as shown in Fig. 1, suggests that the deviation from sphericity of the ground state sets in earlier above $Z=82$ [8]. Extending the results towards the more neutron-deficient radon $(Z=86)$ and radium $(Z=88)$ isotopes could confirm this hypothesis [12]. The platinum isotopes with $Z=78$ show a similar early, but less pronounced, onset of deviation from sphericity as the polonium isotopes $[13,14]$.

The band structure of the neutron-deficient even-even polonium isotopes has been studied extensively. The relevant results of these studies are summarized in the energy systematics of ${ }^{190-210}$ Po shown in Fig. 1. Lifetime measurements of ${ }^{194,196} \mathrm{Po}[15,16]$ and inelastic scattering studies of ${ }^{210} \mathrm{Po}$ [17] provided information on reduced transition probabilities. The level structure of the polonium isotopes was interpreted

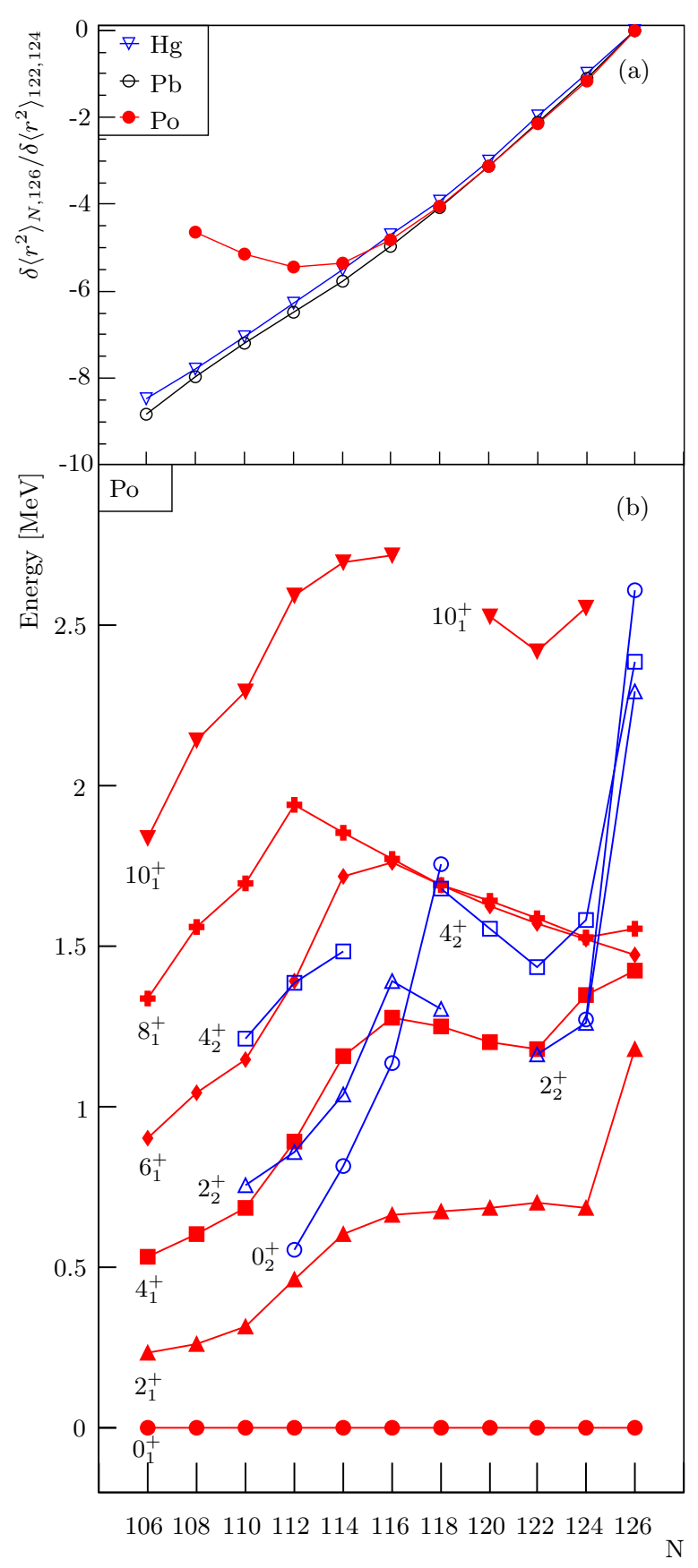

FIG. 1. (Color online) (a) Relative $\delta\left\langle r^{2}\right\rangle$ for the even- $A_{80} \mathrm{Hg}$, ${ }_{82} \mathrm{~Pb}$, and ${ }_{84} \mathrm{Po}$ isotopes [8]. The changes in charge radii, relative to $N=126$, are normalized to the difference in charge radius between $N=122$ and $N=124$. (b) Energy level systematics of the positive-parity states for neutron-deficient even-mass polonium isotopes. Filled (red) symbols show yrast levels; open (blue) symbols, nonyrast levels. Data are taken from Nuclear Data Sheets.

as an anharmonic vibrator in, e.g., [18]. Although vibrational characteristics can be identified in the level systematics of the polonium isotopes, the observation of the downsloping trend of the $0_{2}^{+}$states in ${ }^{196-202} \mathrm{Po}$ is hard to fit into the vibrational picture. Recent literature and theoretical efforts have provided more evidence that points toward the importance of intruder structures $[9,16,19]$. 
Studies within the IBM point out that the energy systematics in platinum isotopes conceal the presence of two different structures, which are reproduced with the inclusion of configuration mixing [20]. Also, in the polonium isotopes, an increasing admixture of deformed configurations in the ground and isomeric states is proposed based on in-beam, $\alpha$-decay, and lifetime studies $[15,21,22]$. Recent beyond-mean-field (BMF) studies of polonium isotopes result in potential-energy surfaces that are soft for heavier polonium isotopes $(\mathrm{A}>198)$, pointing toward the possibility of triaxial structures [19].

Theoretical descriptions, such as phenomenological shapemixing calculations [23-26], contemporary symmetry-guided models [4], and BMF approaches [19], can reproduce the global trends that are deduced from experiments in the lightlead region. However, more subtle experimental information on the nature of the quadrupole deformation and on the mixing between coexisting states is missing for most of the isotopes in the region. Coulomb excitation is a unique tool to study nuclear quadrupole deformation in a model-independent way [27]. It provides access to transitional and diagonal matrix elements, which are good fingerprints for shape coexistence [1]. The recent Coulomb-excitation results on ${ }^{182-188} \mathrm{Hg}$ that were interpreted in the framework of a phenomenological two-level-mixing model provide the first detailed information on mixing in this region [3].

In this paper, we report on two Coulomb-excitation experiments with neutron-deficient ${ }^{196-202}$ Po beams, which were performed at the REX-ISOLDE facility at CERN. Section II reports details on the production and postacceleration of the beams and the specific experimental conditions during the two campaigns. The off-line data analysis is described in detail in Sec. III, while Sec. IV elaborates on the analysis using the Coulomb-excitation analysis code GOSIA. In Sec. V the experimental data are compared to different theoretical nuclear models, and finally, Sec. VI summarizes and formulates conclusions.

\section{EXPERIMENTAL DETAILS}

\section{A. Production, postacceleration, and Coulomb excitation of polonium beams at REX-ISOLDE}

Radioactive ion beams of polonium were produced and postaccelerated at the REX-ISOLDE facility at CERN [28] during two experimental campaigns, in 2009 and 2012. A multitude of isotopes are produced by impinging $1.4-\mathrm{GeV}$ protons, at an average current of $1.6 \mu \mathrm{A}$, on a $\mathrm{UC}_{x}$ target. The produced isotopes diffuse out of the target material, which is kept at a high temperature $\left(T \approx 2000{ }^{\circ} \mathrm{C}\right)$ in order to facilitate the diffusion process and to avoid the sticking of ions to the walls of the target-ion source system. In the RILIS hot cavity, polonium isotopes are resonantly ionized in a three-step laser ionization scheme $[29,30]$. After extraction from the target-ion source system by a $30-\mathrm{kV}$ potential, the desired $1^{+}$-charged isotope is selected by the High-Resolution Separator (HRS). The high temperature of the target-ion source system induces surface ionization of elements with a low ionization potential, giving rise to isobaric contamination from thallium isotopes $(Z=81$, IP $=6.108 \mathrm{eV})$ [31]. The average beam intensities and purities are summarized in Table I. The purity of the beam was extracted based on data acquired when the laser-ON/OFF mode was applied. In this mode the laser ionization is switched periodically on and off using the supercycle of the Proton Synchrotron Booster, with a typical length of $48 \mathrm{~s}$, as the time base for the periodicity. Data acquired in this way contain the same measurement time and conditions with the lasers switched on (thus resonantly ionizing polonium) as with the lasers blocked (only the isobaric contaminant thallium in the beam). A comparison of the number of scattered particles on the particle detector inside the target chamber during the laser$\mathrm{ON}$ and laser-OFF periods of these data, taking into account the difference in Rutherford cross section for polonium $(Z=84)$ and thallium $(Z=81)$, yields the purity of the beam $[32,33]$. On average, the beam purity for ${ }^{198,200,202}$ Po was well above $90 \%$. Only at mass 196 is the $\mathrm{Tl}$ contamination in the beam at the same level as the polonium content.

The low-energetic, isobaric, and singly charged beam, containing the polonium isotope of interest together with the thallium contamination, is then fed into the REX postaccelerator [28]. First, the beam is injected into a Penning trap (REXTRAP) to cool and bunch the continuous beam. The bunches are then charge-bred in the Electron Beam Ion Source (EBIS) to transform $1^{+}$ions to $48^{+}$ions $\left(49^{+}\right.$in the case of ${ }^{202} \mathrm{Po}$ ), with a breeding time of $T=255 \mathrm{~ms}$, resulting in a beam pulse repetition rate of $3.9 \mathrm{~Hz}$. Details on the time structure of the extracted pulse and the way this is treated are given in Ref. [34]. After passing another analyzing magnet the ions are postaccelerated to $2.85 \mathrm{MeV} / u$ by the REX linear accelerator and, finally, delivered to the Miniball detection setup [34].

A secondary thin target (with a thickness of $2.0 \mathrm{mg} / \mathrm{cm}^{2}$ ) is placed in the middle of the Miniball target chamber to induce Coulomb excitation. The beam energy for each

TABLE I. Properties of the beams, associated targets, kinematic characteristics, and running period of the experiments. $I_{\mathrm{Po}, a v}$ represents the average polonium beam intensity measured in the Miniball setup. The purity is defined as the fraction of polonium isotopes in the beam and was determined using scattered particles on the DSSSD during laser-ON/OFF runs. $\theta_{\mathrm{CM}}$ represents the range of center-of-mass scattering angle covered and $T_{\exp }$ is the total measurement time.

\begin{tabular}{lllllll}
\hline \hline$A$ & $T_{1 / 2}(\mathrm{~s})$ & \multicolumn{1}{c}{$I_{\text {Po,av }}(\mathrm{pps})$} & Purity $(\%)$ & Target & $\theta_{\mathrm{CM}}(\mathrm{deg})$ & $T_{\exp }(\mathrm{min})$ \\
\hline 196 & $5.8(2)$ & $2.3(2) \times 10^{4}$ & $59.51(7)$ & ${ }^{104} \mathrm{Pd}$ & $66-128$ & 1687 \\
198 & $106(2)$ & $4.6(7) \times 10^{4}$ & $95.97(19)$ & ${ }^{94} \mathrm{Mo}$ & $66-128$ & 1235 \\
200 & $691(5)$ & $2.54(17) \times 10^{5}$ & $97.90(4)$ & ${ }^{104} \mathrm{Pd}$ & $77-136$ & 2012 \\
202 & $26.8(2) \times 10^{2}$ & $6.6(7) \times 10^{4}$ & $98.3(2)$ & ${ }^{104} \mathrm{Pd}$ & $66-128$ & 196 \\
202 & $26.8(2) \times 10^{2}$ & $4.6(9) \times 10^{4}$ & $98.1(2)$ & ${ }^{94} \mathrm{Mo}$ & $66-128$ & 2012 \\
\hline \hline
\end{tabular}


projectile-target combination was well below the "safe value" ensuring a purely electromagnetic interaction between the colliding nuclei. States up to $4_{1}^{+}$and $2_{2}^{+}$were populated. The choice of the respective target for each isotope (see Table I) was made considering the $\gamma$-ray energies de-exciting the $2_{1}^{+}$ states in the beam and target, to avoid an overlap, and the excitation probability of the target nucleus. The scattered particles are detected with a double-sided-silicon-strip detector (DSSSD), which is also mounted in the target chamber and is divided into 48 secular strips, coupled pairwise and read out by 24 ADC channels, and 16 annular strips to ensure position sensitivity [35]. The distance between target and DSSSD was $32.5 \mathrm{~mm}$ during the experiment in 2009 and $26.5 \mathrm{~mm}$ in 2012, yielding an angular coverage of $15.5^{\circ}<\theta_{\mathrm{LAB}}<$ $51.6^{\circ}$ and $18.8^{\circ}<\theta_{\mathrm{LAB}}<57.1^{\circ}$, respectively. The $\gamma$ rays are detected with the Miniball Ge-detector array that surrounds the target chamber in close geometry. The Miniball detector array consists of eight cluster detectors, of which only seven were operational during both experimental campaigns. Each cluster contains three individually encapsulated hyperpure germanium crystals, which are in turn divided by segmentation of the outer electrode into six segments and a central electrode. The high granularity of the Miniball detectors assures position sensitivity for the $\gamma$-ray detection as well. A combination of ${ }^{152} \mathrm{Eu}$ and ${ }^{133} \mathrm{Ba}$ calibration sources was used to calibrate the energy and to determine the absolute detection efficiency of Miniball over the entire relevant energy range. Caution was paid to the low-energy range so as to ensure a good description of the absolute photon-detection efficiency in the polonium x-ray region [33]. More specifically, relative efficiency curves were normalized to absolute efficiencies using $\gamma \gamma$ coincidences [34].

\section{B. Data taking at Miniball}

The specific timing properties of REX-ISOLDE beams have an implication for the method of data taking at Miniball. As the beam delivered to the REX linear accelerator is bunched, the radio-frequency cavities are not continuously operational. Triggered by the EBIS signal, the linac is switched on during an active window with a length of $800 \mu \mathrm{s}$ and $1 \mathrm{~ms}$ for the 2009 and 2012 experiments, respectively. During the full $800 \mu \mathrm{s} / 1 \mathrm{~ms}$ window, the Miniball data system acquires all the information in the $\gamma$-ray and particle detectors. This window is called the "beam-on" window. To ensure correct identification of all possible sources of background, during an equally long "beam-off" window the data acquisition system is turned on, 4-10 ms after the beam-on window, when no beam is coming from the linear accelerator.

Coincidences between a particle and a $\gamma$ ray (" $p-\gamma$ coincidences") are essential to select the interesting events (Coulomb-excitation events) among the background radiation. The $\gamma$-ray background originates from the room background, decay radiation from the radioactive beam, and $\mathrm{x}$ rays from the accelerator, while the particle background is essentially due to the elastic scattering process. Therefore a specific coincidence scheme is developed for the data system (see Fig. 16 in [34]). An 800-ns-wide coincidence gate is defined about each $\gamma$ ray that is detected in the Miniball array. Particles detected within this window are considered to be coincident with the $\gamma$ ray and treated as $\mathrm{p}-\gamma$ events. In the case of high beam intensities at the Miniball secondary target, particles that do not fall inside the 800-ns coincidence gate can be downscaled. This means that all the coincident particles are registered, but only 1 in $2^{N}$ particles with a $\gamma$ ray outside of the coincidence gate is accepted (with $N$ the downscaling factor), thus reducing the dead time of the particle-detection electronics due to elastically scattered particles. This downscaling method was applied for all polonium isotopes (with downscaling factor $N=4$ ) except for ${ }^{196} \mathrm{Po}$, where the beam intensity was significantly lower (see Table I). However, during the 2012 experimental campaign the $\mathrm{p}-\gamma$ coincidence gate was not set correctly for two of the four quadrants of the DSSSD. This gave rise to downscaling of the $\mathrm{p}-\gamma$ coincidences instead of the particles without coincident $\gamma$ rays for half of the data. The consequences of this incorrect downscaling procedure are discussed in more detail in Sec. III A 2.

\section{OFF-LINE DATA ANALYSIS}

\section{A. Selection of events}

\section{Selection based on kinematic properties}

The Miniball detection setup registers a large number of data on scattered particles and decay radiation. As Coulombexcitation events are hidden in this background, identifying these events of interest is a crucial step in the data analysis. The detected particle energy in the DSSSD as a function of the scattering angle in the laboratory frame of reference $\theta$ for ${ }^{200} \mathrm{Po}$ on ${ }^{104} \mathrm{Pd}$ is shown in Fig. 2. It shows a typical inverse kinematics scattering pattern. The recoiling target atoms (hereafter called "recoils") are scattered throughout the whole detection range of the DSSSD, while the heavier beam particles are detected only at smaller scattering angles in the laboratory frame of reference. It is thus possible to make a distinction between a beam particle and a recoil, based on the energy-versus-angle kinematics. Detected particles in the

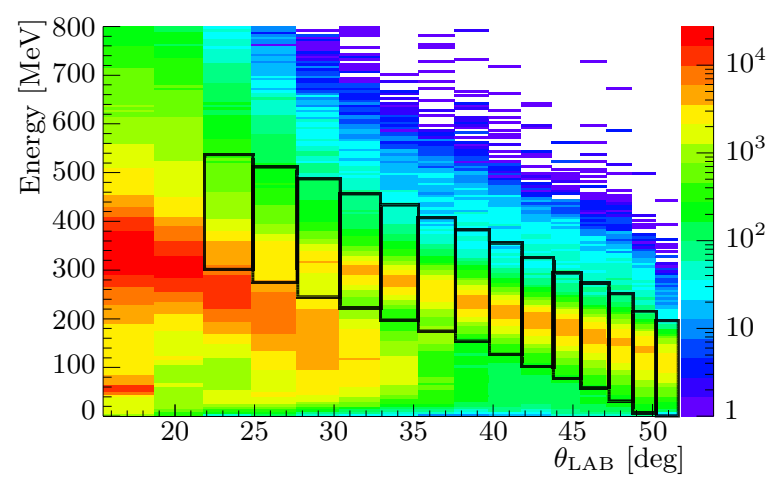

FIG. 2. (Color online) Particle energy versus scattering angle in the laboratory frame of reference $\theta_{\mathrm{LAB}}$ for ${ }^{200} \mathrm{Po}$ on ${ }^{104} \mathrm{Pd}$. The color scale on the vertical axis represents the intensity in each bin. Only particles that are coincident with at least one $\gamma$ ray are shown. Gates chosen to select the ${ }^{104} \mathrm{Pd}$ recoil are shown in black. The two innermost strips are not taken into account, as it is not possible to distinguish between the beam and the recoil particles in this angular range. 

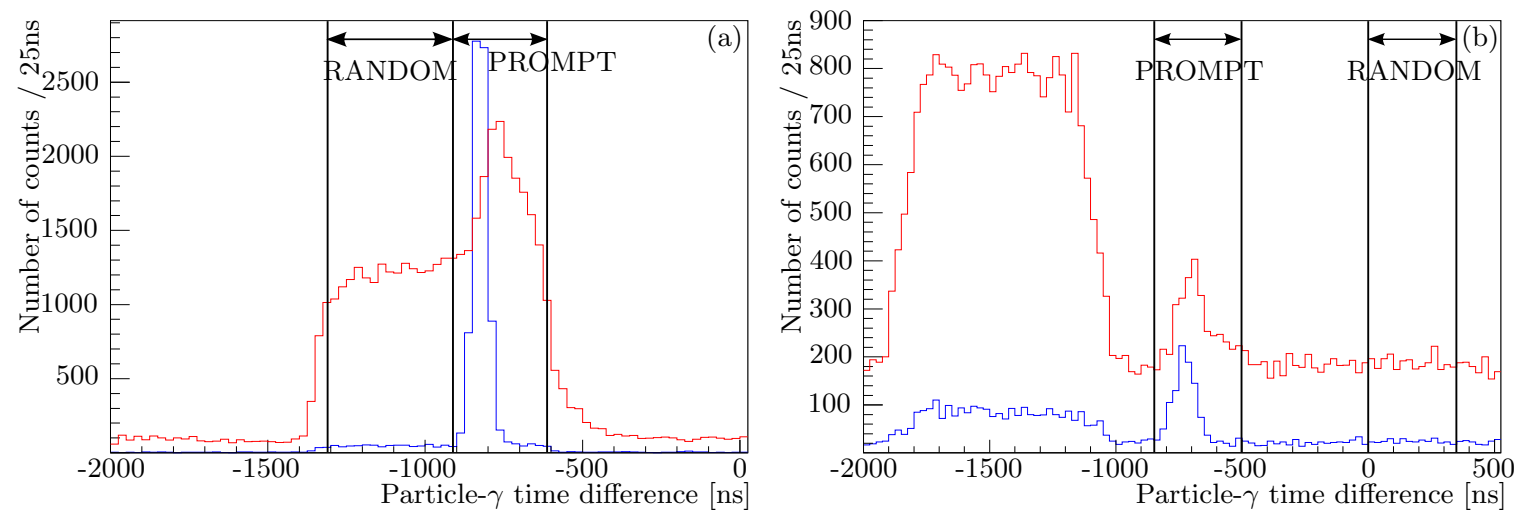

FIG. 3. (Color online) Time difference between particle and $\gamma$ ray, gated on the $2_{1}^{+} \rightarrow 0_{1}^{+} \gamma$-ray transitions (blue lines) and gated on polonium $\mathrm{x}$ rays (red lines). The prompt and random coincidence windows used are shown in black. (a) Sum of $p-\gamma$ timing information for all quadrants (2009 data of ${ }^{200} \mathrm{Po}$ on ${ }^{104} \mathrm{Pd}$ ). (b) $p$ - $\gamma$ timing information for quadrants 1 and 2 where the downscaling was not properly set (2012 data of ${ }^{198} \mathrm{Po}$ on $\left.{ }^{94} \mathrm{Mo}\right)$.

DSSSD related to the scattering of beam on target are selected by "following" the recoils through the range of the DSSSD. For each case, specific $E$-versus- $\theta$ gates were adopted to select the recoils scattered in the particle detector and to avoid including noise into the analysis. As an example, the gates that were used for ${ }^{200} \mathrm{Po}$ on ${ }^{104} \mathrm{Pd}$ can be seen in Fig. 2. The two innermost strips of the particle detector were excluded from the analysis because in this region of the detector the beam and recoil particles are not separable. The range of center-of-mass scattering angles covered by applying this method is listed for each reaction in Table I.

\section{Selection based on timing properties}

Figure 3 shows the time difference between a particle and a $\gamma$ ray detected during the Coulomb-excitation experiments on ${ }^{200}$ Po on ${ }^{104} \mathrm{Pd}$ [Fig. 3(a)] and ${ }^{198}$ Po on ${ }^{94} \mathrm{Mo}$ [Fig. 3(b)]. The different structure of the data on ${ }^{198}$ Po can be explained by a problem with the downscaling in 2012. A difference in time behavior is observed between the $\gamma$ rays following Coulomb excitation (de-exciting the $2_{1}^{+}$state in the polonium isotope) and the low-energy polonium $x$ rays and is due to the energy dependence of the time response of the Ge detectors. The prompt $\mathrm{p}-\gamma$ coincidence window is defined broadly enough to include low-energy $\mathrm{x}$ rays. Random $\mathrm{p}-\gamma$ coincidences are selected with a second time window. In the normal case of the 2009 data on ${ }^{200} \mathrm{Po}$, the random window is chosen within the region where the events are not downscaled. This allows us to scale the prompt and random events using the difference in length of the two respective windows. Data with the wrongly downscaled events were treated in a slightly different way. As the prompt $\mathrm{p}-\gamma$ events fall inside the downscaled region in this case, the random window is also selected among the downscaled events.

The purification power of the event selection based on kinematics and timing is highlighted in Fig. 4, where all detected $\gamma$ rays in the ${ }^{200}$ Po experiment are shown in Fig. 4(a). The $\gamma$ rays following Coulomb excitation are not visible yet in this $\gamma$-ray energy spectrum. By selecting the prompt $\mathrm{p}-\gamma$ coincidences that satisfy the kinematic gates and subsequently subtracting the random coincidences from it, a clean $\gamma$-ray energy spectrum, associated with events following Coulomb excitation, is obtained [Fig. 4(c)]. As the $\gamma$ rays of interest are emitted in flight, the angular information on the detected particle and $\gamma$ ray can be used to perform a Doppler correction of the detected $\gamma$-ray energy. Finally, a $\gamma \gamma$-coincidence window of $350 \mathrm{~ns}$ is defined to check for coincidences between the emitted $\gamma$ rays.

\section{B. Polonium $x$ rays}

In addition to the $\gamma$ rays following the Coulomb excitation of target and projectile, the background-subtracted $\gamma$-ray energy spectra show, for all isotopes studied, two peaks, around 78 and $90 \mathrm{keV}$. These energies correspond to polonium $K_{\alpha}$ and $K_{\beta} \mathrm{x}$ rays, respectively. Origins of these polonium $\mathrm{x}$

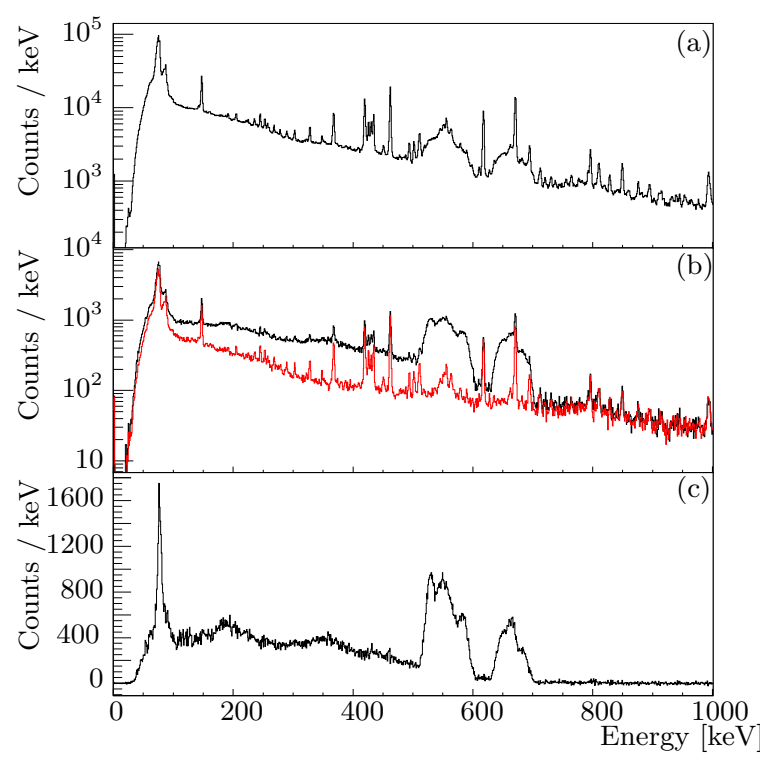

FIG. 4. (Color online) The $\gamma$-ray energy spectra shown here display the 2009 data of ${ }^{200} \mathrm{Po}$ on the ${ }^{104} \mathrm{Pd}$ target. (a) All $\gamma$ rays that were detected. (b) Prompt $\gamma$ rays (black line) and random $\gamma$ rays [gray (red) line] that fulfill the kinematic conditions. (c) Subtracted prompt-minus-random $\gamma$ spectrum. 
TABLE II. The scaling factor to match the predicted and observed amounts of atomically produced $\mathrm{x}$ rays in ${ }^{202,206} \mathrm{Po} . \sigma_{\exp }$ is the experimentally detected $K_{\alpha}$ x-ray cross section related to the atomic effect (corrected for the $\mathrm{x}$ rays attributed to internal conversion), $\sigma_{\text {theo }}$ is the integrated $\mathrm{K}_{\alpha} \mathrm{x}$-ray cross section predicted by theory, and $R$ is the ratio of the observed versus predicted cross section. Conversion coefficients $\alpha_{2_{1}^{+} \rightarrow 0_{1}^{+}}$are taken from [38].

\begin{tabular}{cccclc}
\hline \hline Isotope & Target & $\alpha_{2_{1}^{+} \rightarrow 0_{1}^{+}}$ & $\sigma_{\exp }(\mathrm{b})$ & $\sigma_{\text {theo }}(\mathrm{b})$ & $R$ \\
\hline${ }^{202} \mathrm{Po}$ & ${ }^{104} \mathrm{Pd}$ & $0.01210(17)$ & $0.16(5)$ & $0.743(11)$ & $0.22(6)$ \\
${ }^{202} \mathrm{Po}$ & ${ }^{94} \mathrm{Mo}$ & $0.01210(17)$ & $0.13(4)$ & $0.616(9)$ & $0.21(7)$ \\
${ }^{206} \mathrm{Po}$ & ${ }^{104} \mathrm{Pd}$ & $0.01132(16)$ & $0.15(3)$ & $0.747(11)$ & $0.20(4)$ \\
\hline \hline
\end{tabular}

rays include internal conversion of observed $\gamma$ rays and $E 0$ transitions. An additional source of $\mathrm{x}$ rays that should be taken into account is the heavy-ion-induced $K$-vacancy creation process due to atomic processes in the secondary Coulombexcitation target $[3,36]$. Theoretical formulas describing the cross section for this process as a function of the beam energy, target mass, and ionization potential have to be scaled to match the experimentally observed cross sections. Reference [36] summarizes data on the observation of $\mathrm{x}$ rays in Coulombexcitation experiments on isotopes in the light-lead region at ISOLDE. The cross section for the $K$-vacancy creation process is observed to be significantly higher in the polonium isotopes than in the neighboring isotopes studied (mercury, lead, and radon). In this case, data on the Coulomb excitation of ${ }^{202} \mathrm{Po}$ and ${ }^{206} \mathrm{Po}$ (the latter being part of a different experimental campaign at ISOLDE [37]) were used to scale the theoretical predictions. As no low-lying excited $0^{+}$states are observed in these isotopes, the only nuclear effect giving rise to polonium $\mathrm{x}$ rays is the internal conversion of observed $\gamma$ rays of which the cross section can be calculated using the known conversion coefficients [38]. A weighted-average scaling factor of $0.20(3)$ results from a comparison of the number of observed and expected $\mathrm{x}$ rays (details in Table II).

The scaling factor determined with the data on ${ }^{202,206} \mathrm{Po}$ is then used to rescale the predicted amount of $\mathrm{x}$ rays originating from the heavy-ion-induced $K$-vacancy creation effect for all isotopes. The total number of $\mathrm{x}$ rays is determined using the $K_{\alpha}$ intensity, $K_{\alpha} / K_{\beta}$ branching ratio, and polonium fluorescence yield $\omega_{K}=0.965$ [39]. A comparison of the number of observed $K_{\alpha}$ x rays to the number of (rescaled) expected $K_{\alpha}$ x rays is shown for all studied isotopes in Fig. 5. In the later Coulomb-excitation analysis of the ${ }^{200} \mathrm{Po}$ data the assumption is made that all observed $\mathrm{x}$ rays are related to the atomic effect and the internal conversion of the $2_{1}^{+} \rightarrow 0_{1}^{+}$transition. The limits that can be extracted from the comparison between the number of observed and the number of expected $x$ rays are taken into account in the further analysis for ${ }^{196,198}$ Po. Sections III D 1 and III D 2 describe how $\gamma \gamma$ coincidences are used to distinguish between possible $E 0$ transitions depopulating the $0_{2}^{+}$state and the $2_{2}^{+}$state.

\section{C. ${ }^{94}$ Mo target impurity}

The Coulomb excitation of ${ }^{198}$ Po and ${ }^{202}$ Po was studied using a ${ }^{94}$ Mo target. Based on the energies and transition

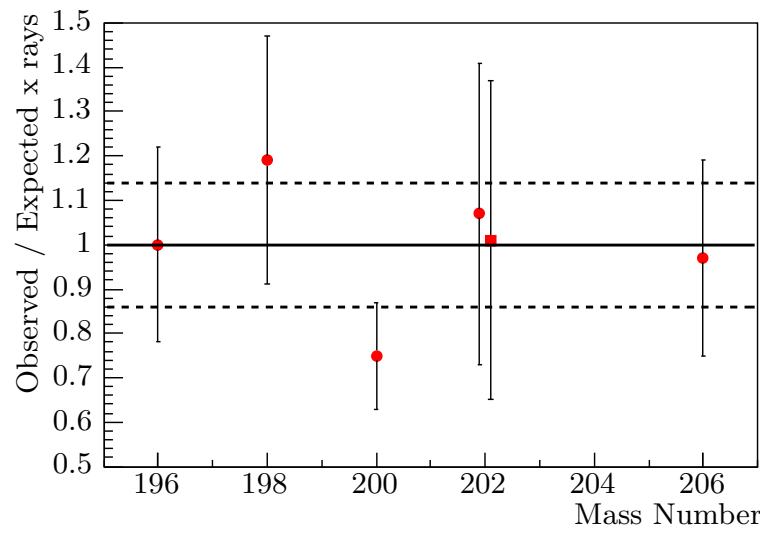

FIG. 5. (Color online) The ratio of the number of observed $x$ rays to the number of (rescaled) expected $\mathrm{x}$ rays is plotted for all studied polonium isotopes. For ${ }^{202} \mathrm{Po}$ two points are drawn: the circle represents the data on ${ }^{104} \mathrm{Pd}$; the square, the data on ${ }^{94} \mathrm{Mo}$ data. The solid horizontal black line represents the scaling factor with the associated uncertainty (dashed horizontal black lines) deduced from the ${ }^{202,206}$ Po data.

probabilities of the low-lying excited states in ${ }^{94}$ Mo (Fig. 6) one $\gamma$-ray transition related to target excitation at $871 \mathrm{keV}$ is expected. However, the background-subtracted $\gamma$-ray energy spectrum for ${ }^{198} \mathrm{Po}$ on ${ }^{94} \mathrm{Mo}$ in Fig. 7 shows a second transition around $200 \mathrm{keV}$. This $\gamma$ ray can be associated with Coulomb excitation of the $3 / 2^{+}$state at $204 \mathrm{keV}$ in the ${ }^{95} \mathrm{Mo}$ impurity in the target as the FWHM of the peak decreases when a Doppler correction for the target recoil trajectory is applied to the $\gamma$-ray energies. The isotopic impurity of the target was independently observed in the analysis of other Coulomb-excitation experiments that used the same target [40,41].

Using the efficiency-corrected intensity balance between the $871-\mathrm{keV}$ and the $204-\mathrm{keV} \gamma$ rays and the Coulombexcitation cross section for ${ }^{94} \mathrm{Mo}$ and ${ }^{95} \mathrm{Mo}$ by a ${ }^{198} \mathrm{Po}$ projectile, the ${ }^{94}$ Mo component in the target was determined to be $F_{94} \mathrm{Mo}=95(2) \%$. As the absolute Coulomb-excitation cross sections in the polonium isotopes are determined by normalization to the known Coulomb-excitation cross section
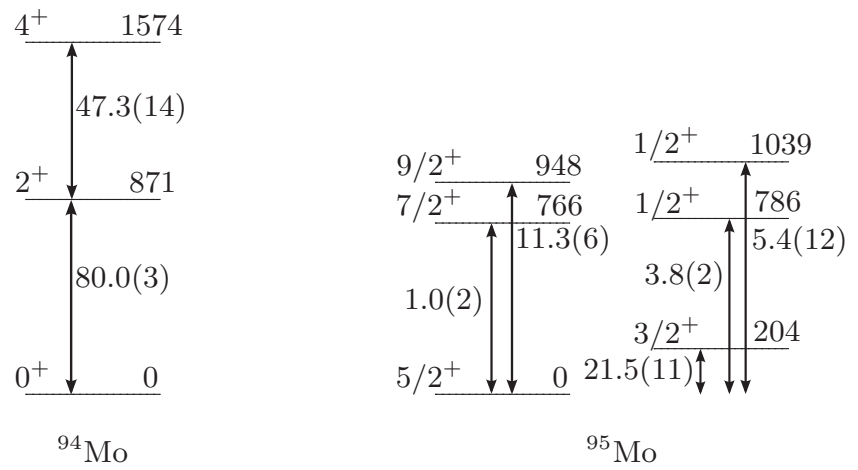

FIG. 6. Level energies (in keV) and reduced transition probabilities $B(E 2) \uparrow$ (in W.u.) of low-lying excited states in ${ }^{94,95}$ Mo. Data are taken from Nuclear Data Sheets and Refs. [42] and [43]. 


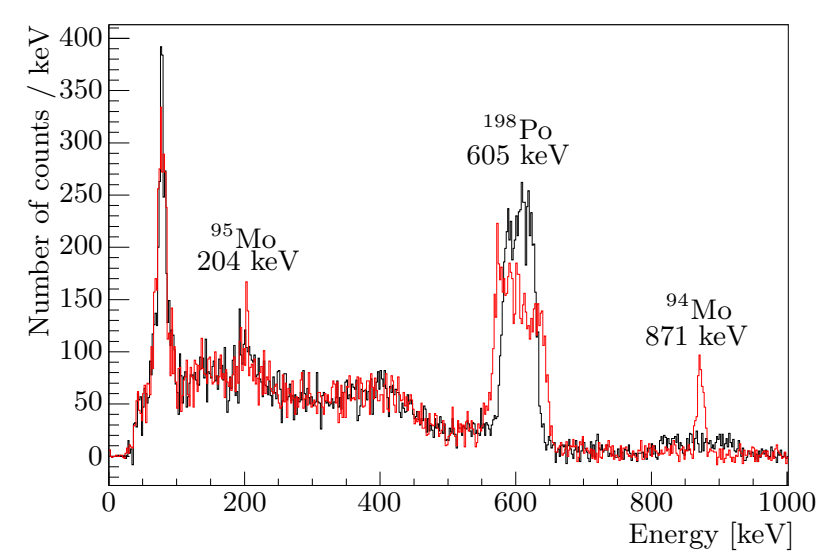

FIG. 7. (Color online) Background-subtracted $\gamma$-ray energy spectrum for ${ }^{198} \mathrm{Po}$ on ${ }^{94} \mathrm{Mo}$ (black line) and Doppler corrected for the target [gray (red) line].

for the given target nucleus (as described in Sec. IV), the target impurity needs to be taken into account. This is done in an indirect way by correcting the number of target excitation counts,

$$
N_{\gamma,{ }^{94} \mathrm{Mo}, \text { total }}=N_{\gamma,{ }^{94} \mathrm{Mo}}\left(1+\frac{F_{95} \mathrm{Mo}}{F_{94} \mathrm{Mo}} \frac{\sigma_{p}\left(Z, A^{\prime}\right)}{\sigma_{p}(Z, A)}\right),
$$

where $F_{94(95)}$ Mo is the fraction of ${ }^{94(95)}$ Mo in the target, $N_{\gamma},{ }^{94} \mathrm{Mo}$ is the number of counts in the $871-\mathrm{keV}$ peak, and $N_{\gamma,{ }^{94} \mathrm{Mo} \text {,total }}$ is the corrected number of ${ }^{94} \mathrm{Mo}$ de-excitations. $\frac{\sigma_{p}\left(Z, A^{\prime}\right)}{\sigma_{p}(Z, A)}$ is the ratio of the cross section for Coulomb excitation of the state of interest in the polonium projectile, incident on a target with mass $A^{\prime}=95$, to the Coulomb-excitation cross section of the state of interest in the polonium projectile, incident on a target with mass $A=94$. This ratio contains the difference in Rutherford cross section and the different center-of-mass energy at different target masses. In the case of ${ }^{202} \mathrm{Po}$, this ratio of cross sections was determined using the projectile matrix elements that were determined with the ${ }^{104} \mathrm{Pd}$ target. In ${ }^{198} \mathrm{Po}$, however, this procedure was not possible, as all the data were taken with the ${ }^{94}$ Mo target. Therefore, the known ratio of Coulombexcitation cross sections of the target (calculated with mass 94 and mass 95) was used as a first-order estimate [44].

\section{Experimental data analysis}

This section describes the data analysis for the four isotopes studied in this work. For each isotope, the backgroundsubtracted and Doppler-corrected $\gamma$-ray energy spectrum, following the Coulomb excitation of the polonium isotope, is shown. In order to be sensitive to the second-order effect of the diagonal matrix element of the $2_{1}^{+}$state, the data are divided into a number of angular ranges. The adopted number of subdivisions per isotope depends on the statistics that were obtained in both the projectile and the target yields. The total statistics that were acquired, together with the deduced Coulomb-excitation cross section $\sigma_{\mathrm{CE}}$, are listed in Table III for all isotopes. The cross section was calculated using the integrated beam current, which was determined using the known cross section for Coulomb excitation of the target
TABLE III. Statistics obtained in the Coulomb-excitation experiments of ${ }^{196-202} \mathrm{Po}$ on the ${ }^{104} \mathrm{Pd}$ and ${ }^{94} \mathrm{Mo}$ targets. $N_{\gamma}$ represents the number of detected $\gamma$ rays at the Miniball setup, and $\sigma_{\mathrm{CE}}$ is the deduced cross section for Coulomb excitation.

\begin{tabular}{|c|c|c|c|}
\hline Nucleus & Transition & $N_{\gamma}$ & $\sigma_{\mathrm{CE}}(\mathrm{b})$ \\
\hline \multicolumn{4}{|c|}{${ }^{202} \mathrm{Po}$ on ${ }^{104} \mathrm{Pd}$} \\
\hline${ }^{202} \mathrm{Po}$ & $2_{1}^{+} \rightarrow 0_{1}^{+}$ & $3.8(3) \times 10^{2}$ & $0.45(6)$ \\
\hline${ }^{104} \mathrm{Pd}$ & $2_{1}^{+} \rightarrow 0_{1}^{+}$ & $1.04(4) \times 10^{3}$ & \\
\hline${ }^{104} \mathrm{Pd}$ & $2_{1}^{+} \rightarrow 0_{1}^{+}$ & $1.04(4) \times 10^{3}$ & \\
\hline \multicolumn{4}{|c|}{${ }^{202}$ Po on ${ }^{94} \mathrm{Mo}$} \\
\hline${ }^{202} \mathrm{Po}$ & $2_{1}^{+} \rightarrow 0_{1}^{+}$ & $2.2(2) \times 10^{2}$ & $0.39(8)$ \\
\hline${ }^{94} \mathrm{Mo}$ & $2_{1}^{+} \rightarrow 0_{1}^{+}$ & $75(13)$ & \\
\hline \multicolumn{4}{|c|}{${ }^{200} \mathrm{Po}$ on ${ }^{104} \mathrm{Pd}$} \\
\hline${ }^{200} \mathrm{Po}$ & $2_{1}^{+} \rightarrow 0_{1}^{+}$ & $1.930(18) \times 10^{4}$ & $0.48(3)$ \\
\hline${ }^{104} \mathrm{Pd}$ & $2_{1}^{+} \rightarrow 0_{1}^{+}$ & $4.37(3) \times 10^{4}$ & \\
\hline \multicolumn{4}{|c|}{${ }^{198}$ Po on ${ }^{94} \mathrm{Mo}$} \\
\hline${ }^{198} \mathrm{Po}$ & $2_{1}^{+} \rightarrow 0_{1}^{+}$ & $4.60(8) \times 10^{3}$ & $1.00(16)$ \\
\hline & $4_{1}^{+} \rightarrow 2_{1}^{+}$ & 171(39) & $0.038(11)$ \\
\hline & $0_{2}^{+} \rightarrow 2_{1}^{+}$ & $78(58)$ & $0.03(4)$ \\
\hline & $2_{2}^{+} \rightarrow 2_{1}^{+}$ & $34(40)$ & $0.010(12)$ \\
\hline${ }^{94} \mathrm{Mo}$ & $2_{1}^{+} \rightarrow 0_{1}^{+}$ & $5.3(3) \times 10^{2}$ & \\
\hline \multicolumn{4}{|c|}{${ }^{196} \mathrm{Po}$ on ${ }^{104} \mathrm{Pd}$} \\
\hline${ }^{196} \mathrm{Po}$ & $2_{1}^{+} \rightarrow 0_{1}^{+}$ & $6.05(9) \times 10^{3}$ & $1.67(19)$ \\
\hline & $4_{1}^{+} \rightarrow 2_{1}^{+}$ & $373(41)$ & $0.108(17)$ \\
\hline & $2_{2}^{+} \rightarrow 0_{1}^{+}$ & $79(12)$ & $0.052(14)$ \\
\hline & $2_{2}^{+} \rightarrow 2_{1}^{+}$ & $85(35)$ & \\
\hline${ }^{104} \mathrm{Pd}$ & $2_{1}^{+} \rightarrow 0_{1}^{+}$ & $5.17(8) \times 10^{3}$ & \\
\hline
\end{tabular}

nucleus, taking into account the beam purity (see Table I), the target purity, and the Miniball detection efficiencies at the respective transition energies [33].

In the two heaviest isotopes studied, only the $2_{1}^{+}$state was populated. The $\gamma$-ray energy spectra are shown in Figs. 8 and 9 for ${ }^{202}$ Po and ${ }^{200}$ Po, respectively. As in ${ }^{196,198}$ Po, multistep Coulomb excitation was observed; additional details related to the data analysis of these two isotopes are provided below.

\section{Data obtained for ${ }^{198}$ Po}

The background-subtracted $\gamma$-ray spectrum of ${ }^{198} \mathrm{Po}$ on ${ }^{94}$ Mo is shown in Fig. 10(a). While in the ${ }^{94}$ Mo target, only the $2_{1}^{+}$state was populated, multiple-step Coulomb excitation was observed in ${ }^{198} \mathrm{Po}$ in the $4_{1}^{+}, 0_{2}^{+}$, and $2_{2}^{+}$states (see level scheme in Fig. 15). A clearer view of the $4_{1}^{+} \rightarrow 2_{1}^{+}$transition results from gating on the $2_{1}^{+} \rightarrow 0_{1}^{+} \gamma$ ray at $605 \mathrm{keV}$ [Fig. 10(b)]. There is only a weak indication of the transitions depopulating the $\mathrm{O}_{2}^{+}$and $2_{2}^{+}$states, which is reflected in the size and relative error of the extracted intensities. The resulting intensities for all the observed transitions, together with the statistics in the $2_{1}^{+} \rightarrow 0_{1}^{+}$transitions in projectile and target, are listed in Table III. The statistics on the $2_{1}^{+} \rightarrow 0_{1}^{+}$ transitions in the projectile and target nuclei allowed us to divide the data into five angular ranges. 


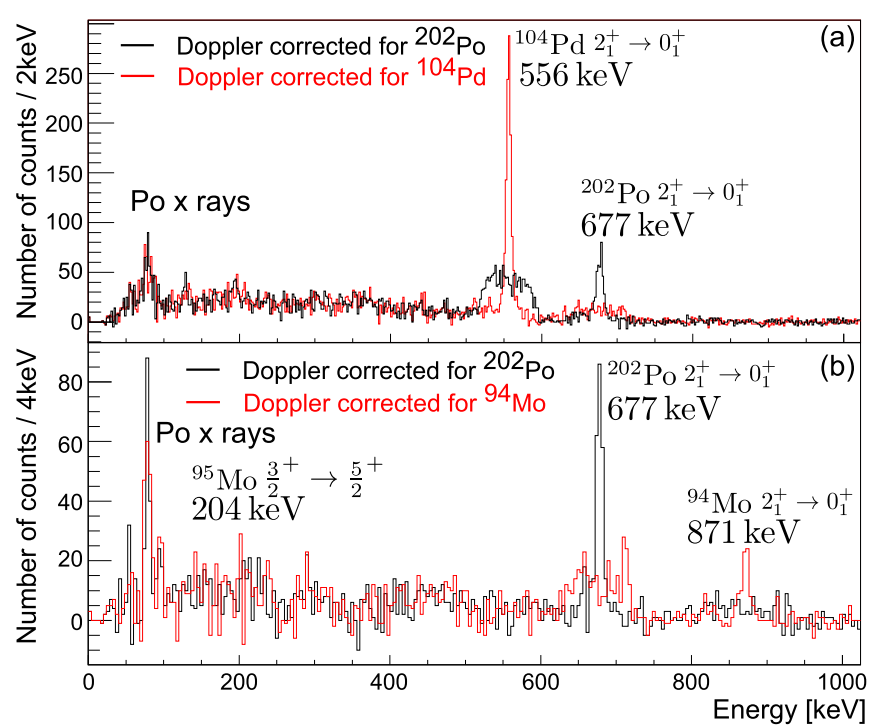

FIG. 8. (Color online) Background-subtracted and Dopplercorrected $\gamma$-ray energy spectrum following the Coulomb excitation of ${ }^{202} \mathrm{Po}$, induced by the ${ }^{202} \mathrm{Po}$ beam impinging (a) on the ${ }^{104} \mathrm{Pd}$ and (b) on the ${ }^{94}$ Mo target. The gray (red) spectrum is Doppler corrected for the target; the black spectrum is Doppler corrected for the projectile. Observed transitions are highlighted.

The particle-gated $\gamma \gamma$-energy spectrum also contains polonium $\mathrm{x}$ rays, which can be attributed to the conversion of observed coincident $\gamma$ rays and to the EO component of the $2_{2}^{+} \rightarrow 2_{1}^{+}$transition. The $4_{1}^{+} \rightarrow 2_{1}^{+}$transition, which is observed both in the "singles" particle-gated $\gamma$-ray energy spectrum and in the particle-gated $\gamma \gamma$ spectrum, is used to link the intensity in the $\gamma \gamma$ spectrum to the singles intensity. A scaling factor $S$ is defined as

$$
S=\frac{I_{4_{1}^{+} \rightarrow 2_{1}^{+}, \mathrm{p} \gamma}}{I_{4_{1}^{+} \rightarrow 2_{1}^{+}, \mathrm{p} \gamma \gamma}}
$$

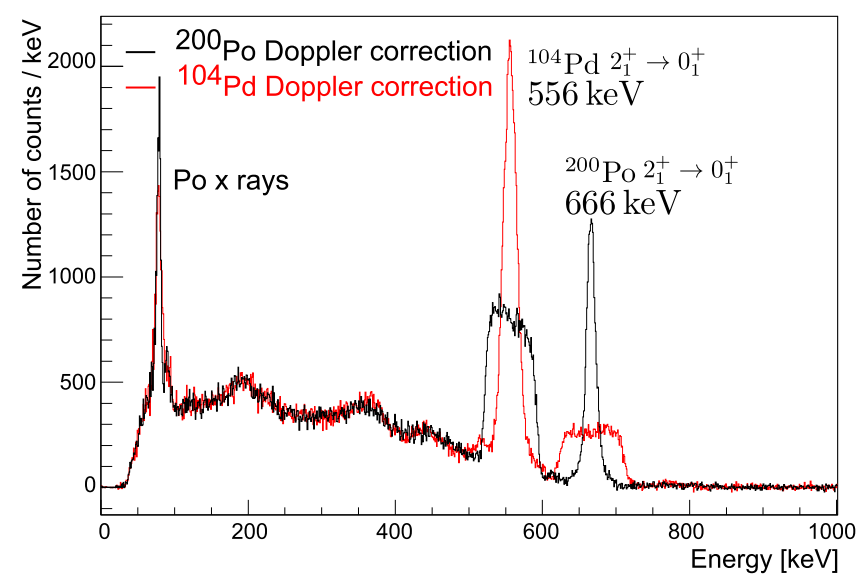

FIG. 9. (Color online) Background-subtracted and Dopplercorrected $\gamma$-ray energy spectrum following the Coulomb excitation of ${ }^{200} \mathrm{Po}$, induced by the ${ }^{200} \mathrm{Po}$ beam impinging on the ${ }^{104} \mathrm{Pd}$ target. The gray (red) spectrum is Doppler corrected for the target; the black spectrum is Doppler corrected for the projectile. Observed transitions are highlighted.

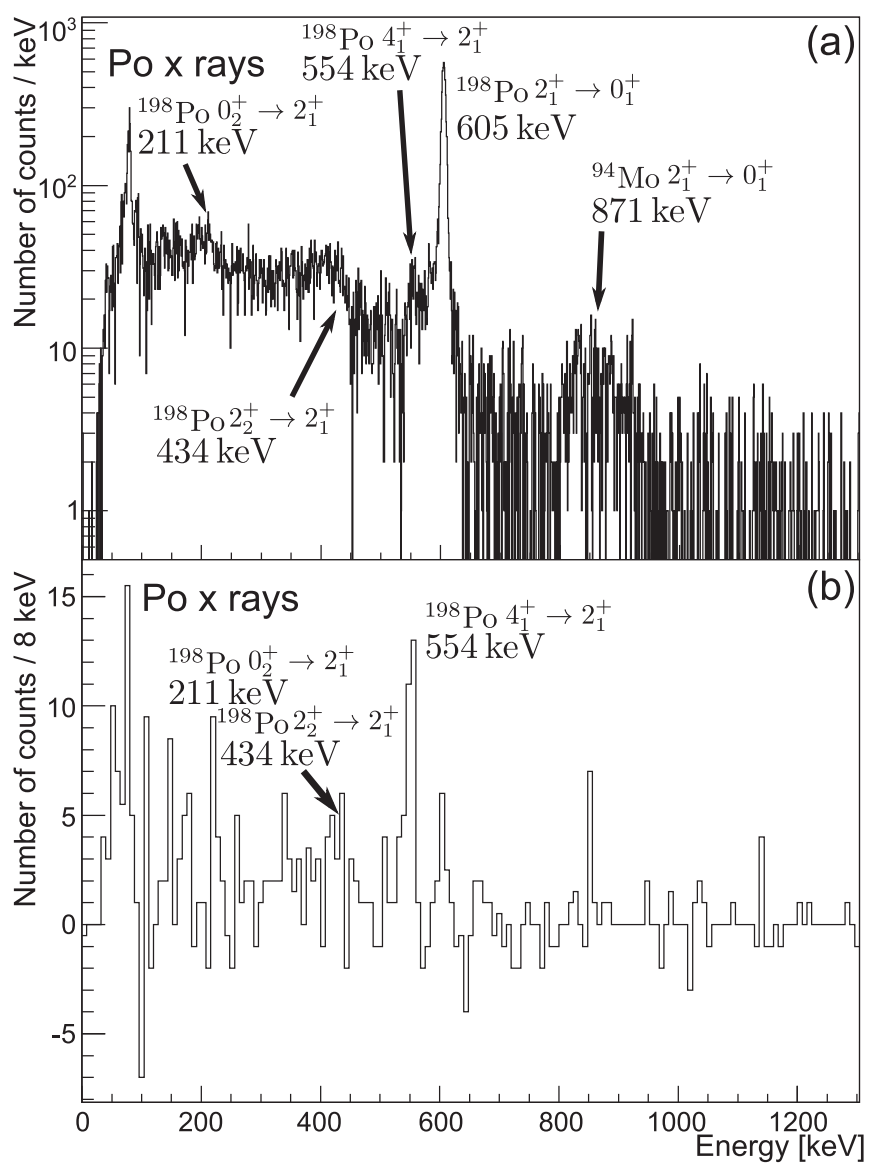

FIG. 10. (a) Background-subtracted and Doppler-corrected $\gamma$-ray energy spectrum following the Coulomb excitation of ${ }^{198} \mathrm{Po}$, induced by the ${ }^{198}$ Po beam on the ${ }^{94}$ Mo target. The $\gamma$-ray energies are Doppler corrected for ${ }^{198} \mathrm{Po}$; the target Doppler correction is shown in Fig. 7. Observed transitions are highlighted. (b) Energy of $\gamma$ rays coincident with the $2_{1}^{+} \rightarrow 0_{1}^{+} \gamma$ ray at $605 \mathrm{keV}$ in ${ }^{198} \mathrm{Po}$. The gated spectrum is background subtracted and Doppler corrected for ${ }^{198} \mathrm{Po}$. Observed transitions in ${ }^{198} \mathrm{Po}$ are highlighted.

which is equal to 10(4) in this case. This factor is mainly determined by the $\gamma$-ray detection efficiency of the $2_{1}^{+} \rightarrow 0_{1}^{+}$ transition and includes the difference in sorting procedure in the construction of the particle-gated $\gamma$ spectrum and the particle-gated $\gamma \gamma$ spectrum [33]. The intensity of the polonium $\mathrm{x}$ rays in the $\gamma \gamma$ spectrum is then corrected for conversion of other $\gamma$-ray transitions in the $\gamma \gamma$ spectrum (assuming $\delta=1.8(5)$ [45]) and for the (small) number of "atomic" $\mathrm{x}$ rays present in the $\gamma \gamma$ spectrum. Of this, a total of 80(130) $\mathrm{x}$ rays is associated with the $E 0$ component of the $2_{2}^{+} \rightarrow 2_{1}^{+}$transition and $150(310) \mathrm{x}$ rays are attributed to the $E 0$ transition between the excited $0^{+}$state and the ground state. Details of this calculation can be found in [33]. The large uncertainties of these numbers are due to the indirect method of determining these intensities.

Finally, following the method described in [46], $1 \sigma$ upper limits were determined for the additional unobserved transitions. The upper limits, listed in Table IV, are taken into account in the Coulomb-excitation analysis described in Sec. IV B 1 . 
TABLE IV. $1 \sigma$ upper limits for unobserved transitions in the Coulomb excitation of ${ }^{198} \mathrm{Po}$ on ${ }^{94} \mathrm{Mo}$. Values for the $1 \sigma$ upper limits (UPL) are determined using the method described in [46] and are not efficiency corrected. The uncertainty of the upper limit represents the $1 \sigma$ uncertainty of the value. $F$ is the efficiency-corrected ratio of the $1 \sigma$ upper limit to the intensity of the $2_{1}^{+} \rightarrow 0_{1}^{+}$transition.

\begin{tabular}{lccc}
\hline \hline Transition & Energy $(\mathrm{keV})$ & UPL & $F$ \\
\hline $6_{1}^{+} \rightarrow 4_{1}^{+}$ & 559 & $44(30)$ & $0.92 \%$ \\
$2_{2}^{+} \rightarrow 0_{2}^{+}$ & 223 & $31(50)$ & $0.40 \%$ \\
$2_{2}^{+} \rightarrow 0_{1}^{+}$ & 1039 & $17(15)$ & $0.48 \%$ \\
\hline \hline
\end{tabular}

\section{Data obtained for ${ }^{196} \mathrm{Po}$}

The Coulomb excitation of ${ }^{196} \mathrm{Po}$ was studied on a ${ }^{104} \mathrm{Pd}$ target. The background-subtracted $\gamma$-ray energy spectrum in Fig. 11(a) shows that multistep Coulomb excitation was observed. The $\gamma$ rays de-exciting the $4_{1}^{+}$and $2_{2}^{+}$states are

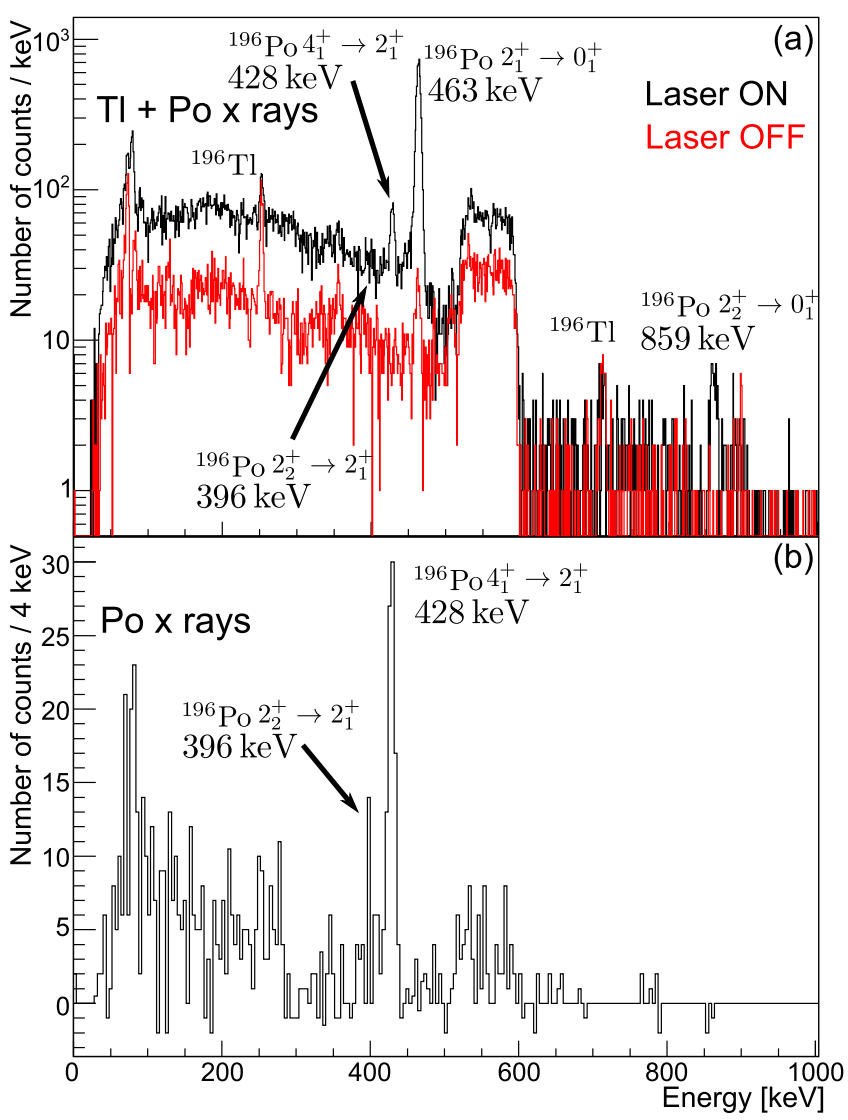

FIG. 11. (Color online) (a) Background-subtracted and Dopplercorrected $\gamma$-ray energy spectrum following the Coulomb excitation of ${ }^{196} \mathrm{Po}$, induced by the ${ }^{196} \mathrm{Po}$ beam on the ${ }^{104} \mathrm{Pd}$ target during equally long laser-ON (black spectrum) and laser-OFF [gray (red) spectrum] windows. The $\gamma$ energies are Doppler corrected for mass 196 and observed transitions are highlighted. The broad structure around $550 \mathrm{keV}$ is due to the ${ }^{104} \mathrm{Pd}$ target excitation at $556 \mathrm{keV}$. (b) Energy of $\gamma$ rays coincident with the $2_{1}^{+} \rightarrow 0_{1}^{+} \gamma$ ray at $463 \mathrm{keV}$ in ${ }^{196} \mathrm{Po}$. The gated spectrum is background subtracted and Doppler corrected for ${ }^{196} \mathrm{Po}$. Observed transitions in ${ }^{196} \mathrm{Po}$ are highlighted. certainly visible next to some lines that cannot be placed in the level scheme of ${ }^{196}$ Po (Fig. 15). The comparison of the Coulomb-excitation spectra, which were acquired during the laser-ON and laser-OFF periods of the laser-ON/OFF data in Fig. 11(a), shows that the unknown transitions originate from de-excitation of populated levels in the isobaric contaminant ${ }^{196} \mathrm{Tl}$.

The beam purity, time-integrated over all the laser-ON/OFF runs, was determined to be 59.51(7)\%. The same method as in [32] was applied to extract the beam purity during the runs where the lasers were on continuously. In this approach, the intensity of the $\gamma$ rays associated with Coulomb excitation of polonium $\left(2_{1}^{+} \rightarrow 0_{1}^{+}\right.$at $\left.463 \mathrm{keV}\right)$ and thallium $\left(1^{-} \rightarrow 2^{-}\right.$at $\left.253 \mathrm{keV}\right)$ were taken into account, together with an extrapolation factor from the laser-ON/OFF runs, yielding a total purity of $46\left({ }_{4}^{6}\right) \%$. The larger relative error bar is due to the smaller statistics in the Coulomb-excitation transitions compared to the scattered particles on the DSSSD. The target de-excitation $\gamma$-ray yields were extracted in a separate analysis for $\mathrm{ON} / \mathrm{OFF}$ runs and $\mathrm{ON}$ runs, employing the respective correction factors for the beam purity.

The projection of the $\gamma \gamma$-energy matrix with a gate on the 463-keV $2_{1}^{+} \rightarrow 0_{1}^{+}$transition is shown in Fig. 11(b). Of the 15(9) detected coincident $K_{\alpha}$ x rays, 4(2) are associated with $E 2 / M 1$ conversion. The unknown E2/M1 mixing ratio of the $2_{2}^{+} \rightarrow 2_{1}^{+}$transition was taken into account by applying the same method as in the case of ${ }^{198}$ Po. The remaining 11(9) $K_{\alpha}$ x rays translate, using Eq. (2) to get to a scaling factor of $S=5.3(9)$, into 76(66) x rays that can be related to the $E 0$ component of the $2_{2}^{+} \rightarrow 2_{1}^{+}$transition. The number of $\mathrm{x}$ rays originating from the $0_{2}^{+} \rightarrow 0_{1}^{+} E 0$ transition is then, finally, determined by subtracting the $K_{\alpha}$ x rays related to internal conversion [370(70)] and the estimate for the $K_{\alpha} \mathrm{x}$ rays originating from the $K$-vacancy creation effect [700(130)] from the total number of detected $K_{\alpha}$ x rays [990(80)]. The calculated $\mathrm{EOO}_{2}^{+} \rightarrow \mathrm{O}_{1}^{+}$intensity is compatible with 0 and gives an upper limit of 140 counts. The detection of $E 00_{2}^{+} \rightarrow 0_{1}^{+}$transitions thus cannot be excluded.

Table III summarizes the intensities of the observed transitions in the Coulomb excitation of ${ }^{196} \mathrm{Po}$ on ${ }^{104} \mathrm{Pd}$. Seven angular ranges were defined for the $2_{1}^{+} \rightarrow 0_{1}^{+}$transitions in target and projectile and for the $4_{1}^{+} \rightarrow 2_{1}^{+}$transition in ${ }^{196} \mathrm{Po}$ to gain sensitivity to second-order effects. The deduced cross section for Coulomb excitation, $\sigma_{\mathrm{CE}}$, is extracted based on the known cross section for Coulomb excitation of the target and taking into account the beam purity and the Miniball detection efficiencies at the respective transition energies. Finally, following the same method as in ${ }^{198} \mathrm{Po}, 1 \sigma$ upper limits were determined for the unobserved transitions (see Table V).

\section{GOSIA ANALYSIS}

The unknown matrix elements coupling low-lying states in polonium isotopes are extracted using the coupled-channels Coulomb-excitation analysis code GOSIA [47,48]. Two approaches are used, depending on the number of states that are populated in the experiment. In the case where only the $2_{1}^{+}$state is populated, GOSIA2 is used (see Sec. IV A). When multistep 
TABLE V. $1 \sigma$ upper limits for unobserved transitions in the Coulomb excitation of ${ }^{196} \mathrm{Po}$ on ${ }^{104} \mathrm{Pd}$. The values for the $1 \sigma$ upper limits (UPLs) are determined using the method described in [46] and are not efficiency corrected. The uncertainty of the UPL represents the $1 \sigma$ uncertainty of the value. $F$ is the efficiency-corrected ratio of the $1 \sigma$ UPL to the intensity of the $2_{1}^{+} \rightarrow 0_{1}^{+}$transition.

\begin{tabular}{lccc}
\hline \hline Transition & Energy $(\mathrm{keV})$ & UPL & $F$ \\
\hline $6_{1}^{+} \rightarrow 4_{1}^{+}$ & 499 & $9(20)$ & $0.2 \%$ \\
$0_{2}^{+} \rightarrow 2_{1}^{+}$ & 95 & $61(70)$ & $0.6 \%$ \\
$2_{2}^{+} \rightarrow 0_{2}^{+}$ & 301 & $48(60)$ & $0.6 \%$ \\
$4_{2}^{+} \rightarrow 4_{1}^{+}$ & 497 & $21(20)$ & $0.4 \%$ \\
$4_{2}^{+} \rightarrow 2_{2}^{+}$ & 529 & $528(50)$ & $9.5 \%$ \\
$4_{2}^{+} \rightarrow 2_{1}^{+}$ & 925 & $8(2)$ & $0.2 \%$ \\
\hline \hline
\end{tabular}

Coulomb excitation is observed, a combined approach using GOSIA and GOSIA2 is employed, as explained in Sec. IV B.

\section{A. Exclusive population of the $2_{1}^{+}$state}

This section deals with the cases of ${ }^{200,202}$ Po in which only the $2_{1}^{+}$state is populated. The Coulomb-excitation cross section is affected by the matrix element coupling the ground state to the populated $2_{1}^{+}$state $\left\langle 0_{1}^{+}\|E 2\| 2_{1}^{+}\right\rangle$and, to second order, by the diagonal matrix element of the $2_{1}^{+}$ state $\left\langle 2_{1}^{+}\|E 2\| 2_{1}^{+}\right\rangle$. The sensitivity to the second-order effect is determined by the obtained statistics, i.e., the number of subdivisions adopted.

Measuring the intensity of the incoming beam is difficult in a radioactive ion-beam experiment with a low beam energy, as the intensity is very low and can fluctuate. The beam can be contaminated as well. Another normalization method is thus needed. The Coulomb-excitation cross section of the projectile is normalized to the target-excitation cross section, which is calculated using the known matrix elements of the target. Table VI lists the matrix elements coupling the relevant states in the ${ }^{104} \mathrm{Pd}$ and ${ }^{94} \mathrm{Mo}$ targets used.

GOSIA2 is a special version of the GOSIA code that simultaneously minimizes the $\chi^{2}$ function for the projectile and target, thus resulting in a set of normalization constants and projectile matrix elements that best reproduce the experimental

TABLE VI. Matrix elements coupling the relevant states in ${ }^{104} \mathrm{Pd}$ and ${ }^{94} \mathrm{Mo}$. These matrix elements were used to determine the cross section for Coulomb excitation of the target.

\begin{tabular}{lccc}
\hline \hline Isotope & Matrix element & Value (eb) & Ref. No. \\
\hline${ }^{104} \mathrm{Pd}$ & $\left\langle 0_{1}^{+}\|E 2\| 2_{1}^{+}\right\rangle$ & $0.73(2)$ & {$[49]$} \\
& $\left\langle 0_{1}^{+}\|E 2\| 2_{2}^{+}\right\rangle$ & $0.134(7)$ & {$[50]$} \\
& $\left\langle 2_{1}^{+}\|E 2\| 2_{1}^{+}\right\rangle$ & $-0.61(15)$ & {$[51]$} \\
& $\left\langle 2_{1}^{+}\|E 2\| 4_{1}^{+}\right\rangle$ & $1.16(3)$ & {$[50]$} \\
& $\left\langle 2_{1}^{+}\|E 2\| 0_{2}^{+}\right\rangle$ & $0.20(1)$ & {$[50]$} \\
& $\left\langle 2_{1}^{+}\|E 2\| 2_{2}^{+}\right\rangle$ & $0.57(3)$ & {$[50]$} \\
${ }^{94} \mathrm{Mo}$ & $\left\langle 0_{1}^{+}\|E 2\| 2_{1}^{+}\right\rangle$ & $0.451(4)$ & {$[52]$} \\
& $\left\langle 2_{1}^{+}\|E 2\| 2_{1}^{+}\right\rangle$ & $0.17(11)$ & {$[53]$} \\
& $\left\langle 2_{1}^{+}\|E 2\| 4_{1}^{+}\right\rangle$ & $0.78(6)$ & {$[54]$} \\
\hline \hline
\end{tabular}

$\gamma$-ray yields. A drawback of the current version of GOSIA2 is that a proper correlated-error determination is not implemented. As only two parameters are determined, the correlated uncertainties are extracted by constructing a two-dimensional $\chi^{2}$ surface and projecting the $1 \sigma$ contour of the total $\chi^{2}$ surface on the respective axis [44]. In this case the $1 \sigma$ contour is defined as the points at which $\chi_{\min }^{2} \leqslant \chi^{2} \leqslant \chi_{\min }^{2}+1$.

$$
\text { 1. }{ }^{202} \mathrm{Po}
$$

Coulomb excitation of ${ }^{202}$ Po was studied using two targets, ${ }^{94} \mathrm{Mo}$ and ${ }^{104} \mathrm{Pd}$. Most of the statistics, especially on target excitation, were collected on the ${ }^{104} \mathrm{Pd}$ target. The $4_{1}^{+} \rightarrow 2_{1}^{+}$ transition in ${ }^{202}$ Po was not observed above the level of $13 \%$ (5\%) relative to the ${ }^{202} \mathrm{Po} 2_{1}^{+} \rightarrow 0_{1}^{+} \gamma$ ray in the ${ }^{104} \mathrm{Pd}\left({ }^{94} \mathrm{Mo}\right)$ experiment. The higher upper limit for the ${ }^{104} \mathrm{Pd}$ target is due to the overlap of the $4_{1}^{+} \rightarrow 2_{1}^{+} \gamma$-ray energy with the target de-excitation transition energy. In both cases the assumption is made that only the $2_{1}^{+}$state is populated. Figure 12 shows the total $\chi^{2}$ surface constructed, in which $\chi^{2}$ is defined as

$$
\chi^{2}=\chi_{\mathrm{Total},{ }^{94} \mathrm{Mo}}^{2}+\chi_{\mathrm{Total},{ }^{104} \mathrm{Pd}}^{2},
$$

where

$$
\chi_{\text {Total }}^{2}=N_{P}^{\text {data }} \chi_{P, \text { GOSIA }}^{2}+N_{T}^{\text {data }} \chi_{T, \text { GOSIA }}^{2} .
$$

Here, $N_{P}^{\text {data }}$ is the number of data points for the projectile [3(2) for the experiment on $\left.{ }^{104} \mathrm{Pd}\left({ }^{94} \mathrm{Mo}\right)\right]$ and $N_{T}^{\text {data }}$ represents the number of data points for the target [5(4) for $\left.{ }^{104} \mathrm{Pd}\left({ }^{94} \mathrm{Mo}\right)\right]$. The number of data points for the target includes the known matrix elements provided to GOSIA (with their error bars). $\chi_{P, \mathrm{GOSIA}}^{2}$ and $\chi_{T, \mathrm{GOSIA}}^{2}$ are the reduced $\chi^{2}$ values given as output by the GOSIA code.

The correlated uncertainties of the transitional and diagonal matrix element can be deduced from the $1 \sigma$ contour as shown in Fig. 12. The resulting matrix elements with their corresponding error bars are listed in Table VII. The value for the transitional matrix element, assuming that $\left\langle 2_{1}^{+} \| E 2|| 2_{1}^{+}\right\rangle=$ $0 \mathrm{eb}$, i.e., with no influence of second-order effects, is also given. The error bar extracted in this way represents the quality

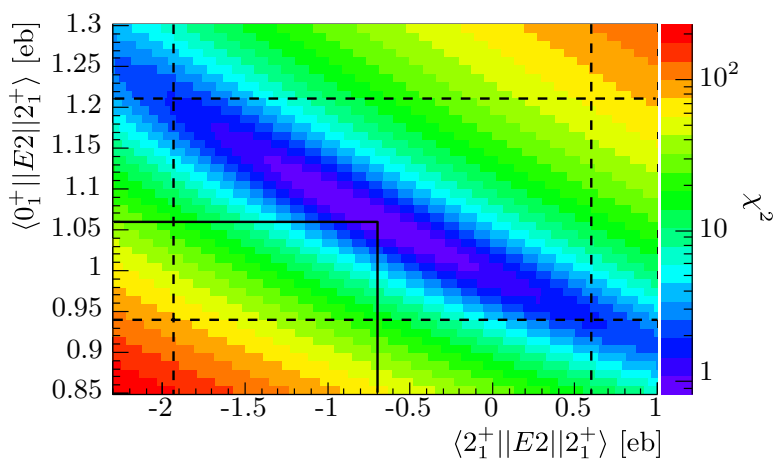

FIG. 12. (Color online) $\chi^{2}$ surface of the transitional and diagonal matrix element of the $2_{1}^{+}$state in ${ }^{202} \mathrm{Po}$. The $\chi^{2}$ is the sum of the $\chi^{2}$ resulting from the experiment on ${ }^{104} \mathrm{Pd}$ and the $\chi^{2}$ extracted from the ${ }^{94}$ Mo experiment. Projection of the $1 \sigma$ contour (dashed lines) gives the correlated uncertainties of the two parameters extracted (see Table VII). 
TABLE VII. Final results for the matrix elements extracted in ${ }^{200,202}$ Po in this work, using the GOSIA 2 code. Two values of the transitional matrix element $\left\langle 0_{1}^{+} \| E 2|| 2_{1}^{+}\right\rangle$are listed: the first result is from the full $\chi^{2}$ surface analysis, and the second value $\left\langle 0_{1}^{+}\|E 2\| 2_{1}^{+}\right\rangle(Q=0)$ is the value that results from projecting the surface at $\left\langle 2_{1}^{+} \| E 2|| 2_{1}^{+}\right\rangle=0 \mathrm{eb}$. Transition energies $E_{\gamma}\left(2_{1}^{+} \rightarrow 0_{1}^{+}\right)$and their uncertainties are taken from the literature.

\begin{tabular}{lccccc}
\hline \hline Isotope & $\begin{array}{c}E_{\gamma}\left(2_{1}^{+} \rightarrow 0_{1}^{+}\right) \\
(\mathrm{keV})\end{array}$ & $\begin{array}{c}\left\langle 0_{1}^{+}\|E 2\| 2_{1}^{+}\right\rangle \\
(\mathrm{eb})\end{array}$ & $\begin{array}{c}\left\langle 2_{1}^{+}\|E 2\| 2_{1}^{+}\right\rangle \\
(\mathrm{eb})\end{array}$ & $\chi_{\min }^{2}$ & $\begin{array}{c}\left\langle 0_{1}^{+}\|E 2\| 2_{1}^{+}\right\rangle(Q=0) \\
(\mathrm{eb})\end{array}$ \\
\hline${ }^{202} \mathrm{Po}$ & $677.2(2)$ & $1.06\left(\begin{array}{c}15 \\
13\end{array}\right)$ & $-0.7\left(\begin{array}{c}13 \\
12\end{array}\right)$ & 0.8 & $0.99(4)$ \\
${ }^{200} \mathrm{Po}$ & $665.9(1)$ & $1.03(3)$ & $0.1(2)$ & 7.9 & $1.040(8)$ \\
\hline \hline
\end{tabular}

of the data in a simplified way and reflects the statistical error of the measured (projectile and target) $\gamma$-ray yields, the uncertainty of the $\gamma$-ray detection efficiency and of the beam and target purity, and the error bar on the matrix elements of the target.

\section{2. ${ }^{200} \mathrm{Po}$}

Coulomb excitation of ${ }^{200}$ Po was studied only with the ${ }^{104} \mathrm{Pd}$ target. The $4_{1}^{+} \rightarrow 2_{1}^{+}$and $0_{2}^{+} \rightarrow 2_{1}^{+} \gamma$ rays were not observed above the level of $0.9 \%$ and $0.7 \%$ relative to the ${ }^{200} \mathrm{Po} 2_{1}^{+} \rightarrow 0_{1}^{+} \gamma$ ray, respectively, so an exclusive population of the $2_{1}^{+}$state was assumed. Figure 13 shows the total $\chi^{2}$ surface constructed applying the $\chi^{2}$ definition given in Eq. (4) with $N_{P}^{\text {data }}=14$ and $N_{T}^{\text {data }}=16$. A significantly higher sensitivity to the second-order effect of the diagonal matrix element results from the large statistics acquired, which allowed us to divide the data into 14 angular ranges. The resulting matrix elements with their corresponding error bars are listed in Table VII. An independent $\chi^{2}$-surface analysis, with 6 angular ranges instead of 14, yielded consistent results with a slightly larger error bar for the $\left\langle 2_{1}^{+}\|E 2\| 2_{1}^{+}\right\rangle$diagonal matrix element. Also, a value for the transitional matrix element, under the assumption that $\left\langle 2_{1}^{+}\|E 2\| 2_{1}^{+}\right\rangle=0$ eb, i.e., with no influence of second-order effects, is given.

\section{B. Population of several low-lying excited states}

In the case of multistep Coulomb excitation to states above the $2_{1}^{+}$state, a combined approach between the standard

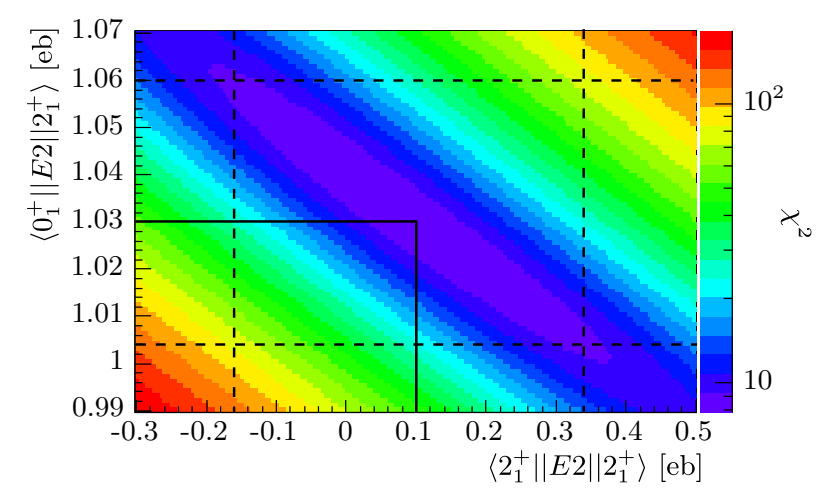

FIG. 13. (Color online) Large-scale $\chi^{2}$ surface of the transitional and diagonal matrix elements of the $2_{1}^{+}$state in ${ }^{200} \mathrm{Po}$. Projection of the $1 \sigma$ contour gives the correlated uncertainties of the two parameters extracted (see Table VII). version of the GOSIA code and GOSIA2 is implemented. The strategy combines the ability to simultaneously minimize the target and projectile $\chi^{2}$ in GOSIA2 and the correlated-error determination of GOSIA. The GOSIA $2 \chi^{2}$-surface analysis is provided as a first approximation in which the influence of higher-order excitations is not considered. The first-order solution for $\left\langle 0_{1}^{+}\|E 2\| 2_{1}^{+}\right\rangle$is used as absolute normalization in the second step, in which GOSIA is used to include couplings to higher-lying excited states. All populated states, observed $\gamma$-ray yields, and relevant spectroscopic data are included, and additionally, a number of "buffer" states are added to avoid artificial population buildup on top of the highest observed state. Including an $E 0$ decay path in GOSIA has to be done in an indirect way by simulating the electron decay via a $M 1$ transition [55]. Thus, nonexisting, additional $1^{+}$states are included in the level scheme of the polonium isotope that take care of the $E 0$ decay paths of the $0_{2}^{+}$and $2_{2}^{+}$states. As $M 1$ excitation is orders of magnitudes weaker than $E 2$ excitation, the $0_{2}^{+}$and $2_{2}^{+}$states are not populated via the $1^{+}$ states. In GOSIA the target de-excitation yields are used to determine relative normalization constants, which are related to the incoming beam intensity and the particle detection efficiency and link the different experimental subdivisions of the data to each other. The solution that results from the GOSIA $\chi^{2}$ minimization is then fed again to GOSIA 2 to check the stability of the solution for $\left\langle 0_{1}^{+}\|E 2\| 2_{1}^{+}\right\rangle$. In this step the couplings between states above the $2_{1}^{+}$state are fixed and only $\left\langle 0_{1}^{+}\|E 2\| 2_{1}^{+}\right\rangle$and $\left\langle 2_{1}^{+}\|E 2\| 2_{1}^{+}\right\rangle$are free parameters of the GOSIA 2 fit. Iterations between GOSIA and GOSIA 2 are performed until a consistent solution is reached [44].

\section{1. ${ }^{198} \mathrm{Po}$}

The ${ }^{94}$ Mo target was used to study the Coulomb excitation of ${ }^{198}$ Po. Multistep Coulomb excitation up to the $4_{1}^{+}, 2_{2}^{+}$, and $0_{2}^{+}$states was observed. The first approximation with GOSIA2 yields a minimum at $\chi^{2}=3.9$ for $\left\langle 0_{1}^{+}\|E 2\| 2_{1}^{+}\right\rangle=1.14\left(\begin{array}{l}12 \\ 11\end{array}\right) \mathrm{eb}$ and $\left\langle 2_{1}^{+}\|E 2\| 2_{1}^{+}\right\rangle=3.6\left(\begin{array}{c}17 \\ 14\end{array}\right)$ eb. The first-order solution for $\left\langle 0_{1}^{+}\|E 2\| 2_{1}^{+}\right\rangle$is then used as an additional data point in the GOSIA analysis, together with the known and relevant spectroscopic information on ${ }^{198} \mathrm{Po}$, which is listed in Table VIII. The E2/M1 mixing ratio determined in ${ }^{202} \mathrm{Po}$ is assumed to stay constant for the neighboring polonium isotopes, which is an approximation. However, as the Coulomb-excitation data are insensitive to the $M 1$ component of the mixed $E 2 / M 1$ transitions, this does not influence the extracted matrix elements. 
TABLE VIII. Spectroscopic information on ${ }^{196,198}$ Po included in the GOSIA analysis. The E2/M1 mixing ratio determined in ${ }^{202} \mathrm{Po}$ is assumed to stay constant for the neighboring polonium isotopes. The $I_{2_{2}^{+} \rightarrow 2_{1}^{+}} / I_{2_{2}^{+} \rightarrow 0_{1}^{+}}$branching ratio is the $\gamma$-ray branching ratio and does not include $E 0$ components.

\begin{tabular}{lcc}
\hline \hline Observable & Value & Ref. No. \\
\hline & ${ }^{196} \mathrm{Po}$ & \\
$\tau_{2_{1}^{+}}$ & $11.7(15) \mathrm{ps}$ & {$[16]$} \\
$\tau_{4_{1}^{+}}$ & $7.8(11) \mathrm{ps}$ & {$[16]$} \\
$\tau_{6_{1}^{+}}$ & $2.9(12) \mathrm{ps}$ & {$[16]$} \\
$I_{2} \rightarrow 2_{1}^{+} / I_{2_{2}^{+} \rightarrow 0_{1}^{+}}$ & $0.64(3)$ & {$[18]$} \\
$\delta(E 2 / M 1)$ & $1.8(5)$ & {$[45]$} \\
& $198 \mathrm{Po}$ & \\
$I_{2} \rightarrow 2_{1}^{+} / I_{2_{2}^{+} \rightarrow 0_{1}^{+}}$ & $2.1(11)$ & {$[56]$} \\
$I_{0_{2}^{+} \rightarrow 0_{1}^{+}} / I_{0_{2}^{+} \rightarrow 2_{1}^{+}}$ & $2.2(16)$ & {$[57]$} \\
$\delta(E 2 / M 1)$ & $1.8(5)$ & {$[45]$} \\
\hline \hline
\end{tabular}

Next to the populated states, the $6_{1}^{+}$and $4_{2}^{+}$states were included as buffer states. The $E 0$-decay transitions of the $0_{2}^{+}$ and $2_{2}^{+}$states were simulated via $M 1$ transitions through two $1^{+}$states included in the level scheme at 300 and at $700 \mathrm{keV}$. A $\chi^{2}$ minimization is performed resulting in four sets of matrix elements that reproduce the experimental data on a comparable level. The four solutions represent four different relative sign combinations for the matrix elements. Solutions 1 and 2, listed in Table IX, represent two solutions where $\left\langle 0_{1}^{+} \| E 2|| 2_{2}^{+}\right\rangle$is positive. The solutions where $\left\langle 0_{1}^{+}\|E 2\| 2_{2}^{+}\right\rangle$ is negative (solutions 3 and 4) are not listed in Table IX, as they are not considered to be physical solutions. The relative signs of the matrix elements affect the Coulomb-excitation cross section in an important way. Every possible excitation path contributes to the cross section for multistep Coulomb

TABLE IX. Two sets of reduced transitional and diagonal E2 matrix elements between low-lying states in ${ }^{196,198}$ Po obtained in this work. Error bars correspond to $1 \sigma$. The different solutions correspond to different relative sign combinations of the matrix elements.

\begin{tabular}{|c|c|c|}
\hline$\left\langle I_{i} \| E 2|| I_{f}\right\rangle(\mathrm{eb})$ & Solution 1 & Solution 2 \\
\hline \multicolumn{3}{|c|}{${ }^{196} \mathrm{Po}$} \\
\hline$\left\langle 0_{1}^{+}\|E 2\| 2_{1}^{+}\right\rangle$ & $1.32(5)$ & $1.32(5)$ \\
\hline$\left\langle 0_{1}^{+}\|E 2\| 2_{2}^{+}\right\rangle$ & $0.44(4)$ & $-0.44(3)$ \\
\hline$\left\langle 2_{1}^{+}\|E 2\| 2_{1}^{+}\right\rangle$ & $-0.2(4)$ & $1.1(5)$ \\
\hline$\left\langle 2_{1}^{+}\|E 2\| 2_{2}^{+}\right\rangle$ & $2.12\left(\begin{array}{c}16 \\
22\end{array}\right)$ & $2.04\left(\begin{array}{l}15 \\
18\end{array}\right)$ \\
\hline$\left\langle 2_{1}^{+}\|E 2\| 4_{1}^{+}\right\rangle$ & $2.68(11)$ & $2.69\left(\begin{array}{l}12 \\
11\end{array}\right)$ \\
\hline \multicolumn{3}{|c|}{${ }^{198} \mathrm{Po}$} \\
\hline$\left\langle 0_{1}^{+}\|E 2\| 2_{1}^{+}\right\rangle$ & $1.15(13)$ & $1.15(13)$ \\
\hline$\left\langle 0_{1}^{+}\|E 2\| 2_{2}^{+}\right\rangle$ & $0.25\left(\begin{array}{l}11 \\
4\end{array}\right)$ & $0.27\left(\left(_{5}^{10}\right)\right.$ \\
\hline$\left\langle 2_{1}^{+}\|E 2\| 2_{1}^{+}\right\rangle$ & $2.9\left(\begin{array}{l}14 \\
15\end{array}\right)$ & $2.4\left(\begin{array}{l}16 \\
14\end{array}\right)$ \\
\hline$\left\langle 2_{1}^{+}\|E 2\| 0_{2}^{+}\right\rangle$ & $1.4\left(\begin{array}{c}24 \\
7\end{array}\right)$ & $-1.8\left({ }_{19}^{8}\right)$ \\
\hline$\left\langle 2_{1}^{+}\|E 2\| 2_{2}^{+}\right\rangle$ & $2.8(9)$ & $3.1(9)$ \\
\hline$\left\langle 2_{1}^{+}\|E 2\| 4_{1}^{+}\right\rangle$ & $3.3\left({ }_{5}^{4}\right)$ & $3.2(4)$ \\
\hline$\left\langle 0_{2}^{+}\|E 2\| 2_{2}^{+}\right\rangle$ & $1.2(8)$ & $<3(3)$ \\
\hline
\end{tabular}

excitation to a certain excited state. As the excitation amplitude for a given path is proportional to the product of the matrix elements involved, the relative signs of these matrix elements play a crucial role. The signs of the products of matrix elements were varied by carefully adopting various initial values, and all possible sign combinations were investigated $[33,44]$.

In solutions 3 and 4 (not listed in Table IX), the population of the $2_{2}^{+}$state is significantly lower than in solutions 1 and 2. In order to reproduce the experimental yields, the diagonal matrix element of the $2_{1}^{+}$state has to be increased in these solutions to unphysically large values of $>4 \mathrm{eb}$, far beyond the rotational limit. Because of these large values for the diagonal matrix element, solutions 3 and 4 are disregarded. The sign of the loop $\left\langle 2_{1}^{+} \| E 2|| 0_{2}^{+}\right\rangle \cdot\left\langle 0_{2}^{+}\|E 2\| 2_{2}^{+}\right\rangle \cdot\left\langle 2_{2}^{+}\|E 2\| 2_{1}^{+}\right\rangle$ is the only difference between solution 1 and solution 2 , which are listed in Table IX (positive for solution 1, negative for solution 2). Changing the sign of this loop does not change the population of any of the excited states significantly. However the matrix element $\left\langle 0_{2}^{+}\|E 2\| 2_{2}^{+}\right\rangle$reaches the lower limit 0 in solution 2, hinting at the fact that a better solution would be obtained with a negative sign for this matrix element. When the sign of the matrix element between the $0_{2}^{+}$and the $2_{2}^{+}$state is changed, the first solution is reproduced exactly in magnitude, but with a negative value for $\left\langle 2_{1}^{+}\|E 2\| 0_{2}^{+}\right\rangle$and $\left\langle 0_{2}^{+}\|E 2\| 2_{2}^{+}\right\rangle$. However, the positive sign for the $\left\langle 2_{1}^{+}|| E 2 \| 0_{2}^{+}\right\rangle \cdot\left\langle 0_{2}^{+}\|E 2\| 2_{2}^{+}\right\rangle \cdot\left\langle 2_{2}^{+} \| E 2|| 2_{1}^{+}\right\rangle$loop is not changed. This is an argument for putting solution 1 forward as the correct sign combination.

The matrix elements of solution 1 are fixed in a new $\chi^{2}$ analysis in GOSIA2 where only $\left\langle 0_{1}^{+}\|E 2\| 2_{1}^{+}\right\rangle$and $\left\langle 2_{1}^{+}\|E 2\| 2_{1}^{+}\right\rangle$ are allowed to vary. The resulting $1 \sigma$ contour is shown in Fig. 14, yielding a result for both matrix elements which is consistent with the GOSIA minimum $\left[\left\langle 0_{1}^{+}|| E 2|| 2_{1}^{+}\right\rangle=1.14(14) \mathrm{eb}\right.$, $\left.\left\langle 2_{1}^{+}\|E 2\| 2_{1}^{+}\right\rangle=2.4\left(\begin{array}{c}21 \\ 16\end{array}\right) \mathrm{eb}\right]$.

\section{2. ${ }^{196} \mathrm{Po}$}

The Coulomb excitation of the lightest polonium isotope studied in this work, ${ }^{196} \mathrm{Po}$, was examined on a ${ }^{104} \mathrm{Pd}$

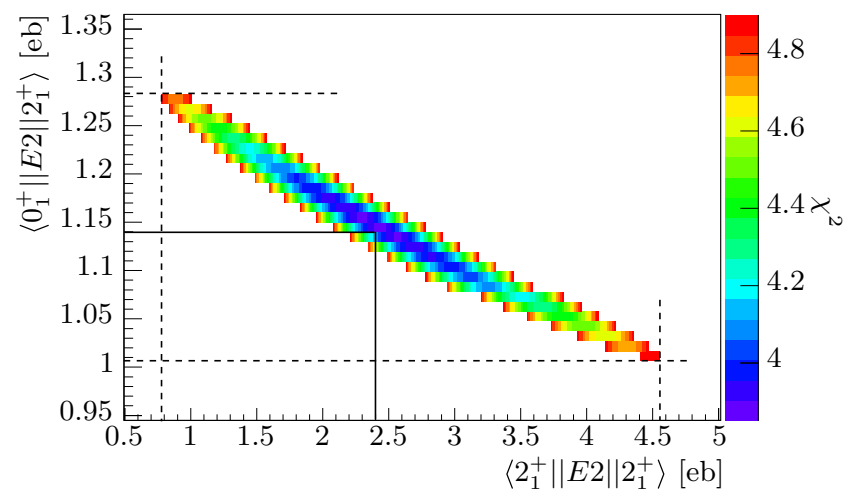

FIG. 14. (Color online) Result of the $\chi^{2}$-surface analysis for the Coulomb excitation of ${ }^{198} \mathrm{Po}$ on ${ }^{94} \mathrm{Mo}$ showing the $1 \sigma$ contour constructed by letting $\chi^{2}$ increase to $\chi_{\min }^{2}+1=4.9$. Projection of the $1 \sigma$ contour gives the correlated uncertainties of the two parameters that are extracted. 
target. Multistep Coulomb excitation to the $4_{1}^{+}, 2_{2}^{+}$, and $0_{2}^{+}$states was observed. The relevant spectroscopic information on ${ }^{196}$ Po included in the GOSIA analysis is listed in Table VIII. The first approximation with GOSIA2, with all the relevant spectroscopic information included, yields a minimum at $\chi_{\min }^{2}=13.1$ for $\left\langle 0_{1}^{+}\|E 2\| 2_{1}^{+}\right\rangle=1.36\left(\begin{array}{c}5 \\ 6\end{array}\right) \mathrm{eb}$ and $\left\langle 2_{1}^{+}\|E 2\| 2_{1}^{+}\right\rangle=0.1\left({ }_{5}^{6}\right) \mathrm{eb}$. The first-order solution for $\left\langle 0_{1}^{+}\|E 2\| 2_{1}^{+}\right\rangle$is used asan additional data point in the GOSIA analysis, together with the relevant spectroscopic information, which is listed in Table VIII. The $E 0$ transitions of the $0_{2}^{+}$and $2_{2}^{+}$states were simulated via $M 1$ transitions through two $1^{+}$ states included in the level scheme at 300 and $650 \mathrm{keV}$. A $\chi^{2}$ minimization, checking also the sensitivity of the signs of the loops of matrix elements, leads to two sets of matrix elements that reproduce the experimental data on a comparable level (see Table IX). A lack of experimental information on the coupling between the $0_{2}^{+}$state and the $2_{1}^{+}$and $2_{2}^{+}$states renders it impossible to extract information on the sign and magnitude of $\left\langle 2_{1}^{+}\|E 2\| 0_{2}^{+}\right\rangle$and $\left\langle 0_{2}^{+}\|E 2\| 2_{2}^{+}\right\rangle$. However, to make sure that the correlations to these couplings are taken into account, the matrix elements were included in the GOSIA analysis, as well as the buffer states $6_{1}^{+}$and $4_{2}^{+}$. It is clear from Table IX that the sign of the loop $\left\langle 0_{1}^{+}\|E 2\| 2_{1}^{+}\right\rangle \cdot\left\langle 2_{1}^{+}\|E 2\| 2_{2}^{+}\right\rangle \cdot\left\langle 2_{2}^{+}\|E 2\| 0_{1}^{+}\right\rangle$ influences only the value of the diagonal matrix element of the $2_{1}^{+}$state significantly. There is no model-independent way to distinguish between these two solutions with the present set of data.

\section{DISCUSSION}

Mixing between coexisting structures has a large influence on the matrix elements and depends strongly on the proximity of energy levels of the same spin. Figure 15 shows systematically both the level energies and the transitional quadrupole moments $\left|Q_{t}\right|$ for ${ }^{196-202}$ Po.

The experimentally obtained results are compared using three theoretical approaches: the BMF method, the generalized Bohr Hamiltonian (GBH), and the IBM. The first two methods are based on the introduction of a mean field determined by the HFB method and the same SLy4 energy density functional. In the BMF method, the mean-field wave functions are first projected on the angular momentum and particle number and then mixed with respect to the axial quadrupole moment. Spectra and transition probabilities are calculated in the laboratory frame of reference and compared directly to the experimental data [19]. In the GBH method, the mass parameters of a Bohr Hamiltonian are derived thanks to a cranking approximation to the adiabatic time-dependent Hartree-Fock method and are rescaled to take into account the fact that time-odd contributions to the mass parameters are neglected. One of the benefits of this method is that it leads to calculations much less heavy than the BMF method and permits the treatment of triaxial quadrupole deformations $[59,60]$. Note that in both methods, the only parameters are those of the energy density functional and no specific adjustments are performed in their applications to the neutrondeficient isotopes around lead. The IBM is a very convenient method to put into evidence the group properties of nuclear spectra and to classify them using group theoretical methods.
However, it contains eight parameters per isotope in the form used here, which are adjusted for each isotope thanks to known experimental data. The measured energies for the yrast band up to $I=8^{+}$, states $0_{2}^{+}, 2_{2}^{+}, 2_{3}^{+}, 2_{4}^{+}, 3_{1}^{+}, 4_{2}^{+}, 4_{3}^{+}, 5_{1}^{+}$, and $6_{2}^{+}$, and the measured $B(E 2)$ values between the above states are used to fix the parameters of the Hamiltonian through a least-squares fit. The purpose of the IBM is therefore to analyze data but it is less suited to perform predictions for unknown nuclei [4].

The BMF approach overestimates the level energies in the four polonium isotopes studied here, as noted by Yao et al. [19]. The level energies in the neighboring mercury, lead, and radon isotopes are also too widely spaced in the BMF calculations. The results obtained using the $\mathrm{GBH}$ approach are significantly better, pointing out the importance of triaxial quadrupole deformations, although the renormalization of the GBH mass parameters does not allow a firm conclusion. The transition probabilities between the ground state and the $2_{1}^{+}$ state are reproduced quite well for ${ }^{200,202} \mathrm{Po}$, suggesting the correct description of the underlying structures. For mass $A<200$, these transition probabilities are underestimated. Further, significant differences can be noted in the transition probabilities related to the $0_{2}^{+}$versus $2_{2}^{+}$state resulting from the three theoretical descriptions. The triaxial quadrupole degree of freedom included in the GBH approach does not significantly affect the transition probabilities.

Figure 16 shows a comparison of extracted deformation parameters obtained from the measured charge radii $\delta\left\langle r^{2}\right\rangle$, on the one hand, and from the sum of squared matrix elements $\sum_{i}\left|\left\langle 0_{1}^{+}|| E 2|| 2_{i}^{+}\right\rangle\right|^{2}$, on the other. As the parameters extracted from these two approaches are not identical, separate notation is used. A deformation parameter, called $\tilde{\beta}_{2}$, was estimated from the charge radii using the expression

$$
\left\langle r^{2}\right\rangle_{A} \approx\left\langle r^{2}\right\rangle_{A}^{\mathrm{sph}}\left(1+\frac{5}{4 \pi} \tilde{\beta}_{2}^{2}\right)
$$

where $\left\langle r^{2}\right\rangle_{A}^{\mathrm{sph}}$ is the mean-square charge radius of a spherical nucleus with the same volume, which was evaluated with the droplet model with a revised parametrization [61]. From the extracted $E 2$ matrix elements, a deformation parameter, $\beta_{2}$, can be deduced, through the quadrupole invariant $\left\langle Q^{2}\right\rangle$, using the expression

$$
\sum_{i}\left|\left\langle 0_{1}^{+}\|E 2\| 2_{i}^{+}\right\rangle\right|^{2}=\left(\frac{3}{4 \pi} Z e R_{0}^{2}\right)^{2} \beta_{2}^{2},
$$

where a uniform charge distribution is assumed [62]. The sum of squared matrix elements $\left|\left\langle 0_{1}^{+}|| E 2|| 2_{i}^{+}\right\rangle\right|^{2}$ was evaluated over the $2^{+}$states populated for each case, i.e., only the $2_{1}^{+}$ state in ${ }^{200,202} \mathrm{Po}$ and the $2_{1}^{+}$and $2_{2}^{+}$states in ${ }^{196,198}$ Po. In ${ }^{194} \mathrm{Po}$, only the $B(E 2)$ value of the $2_{1}^{+}$state is known from the lifetime measurement [15]. The onset of deviation from sphericity around $N=112(A=196)$, observed in the laser spectroscopy studies (see also Fig. 1), is confirmed by the measured transition probabilities. An overall good agreement between the deformation parameter extracted from the charge radii and the squared matrix elements is observed within the error bars. 


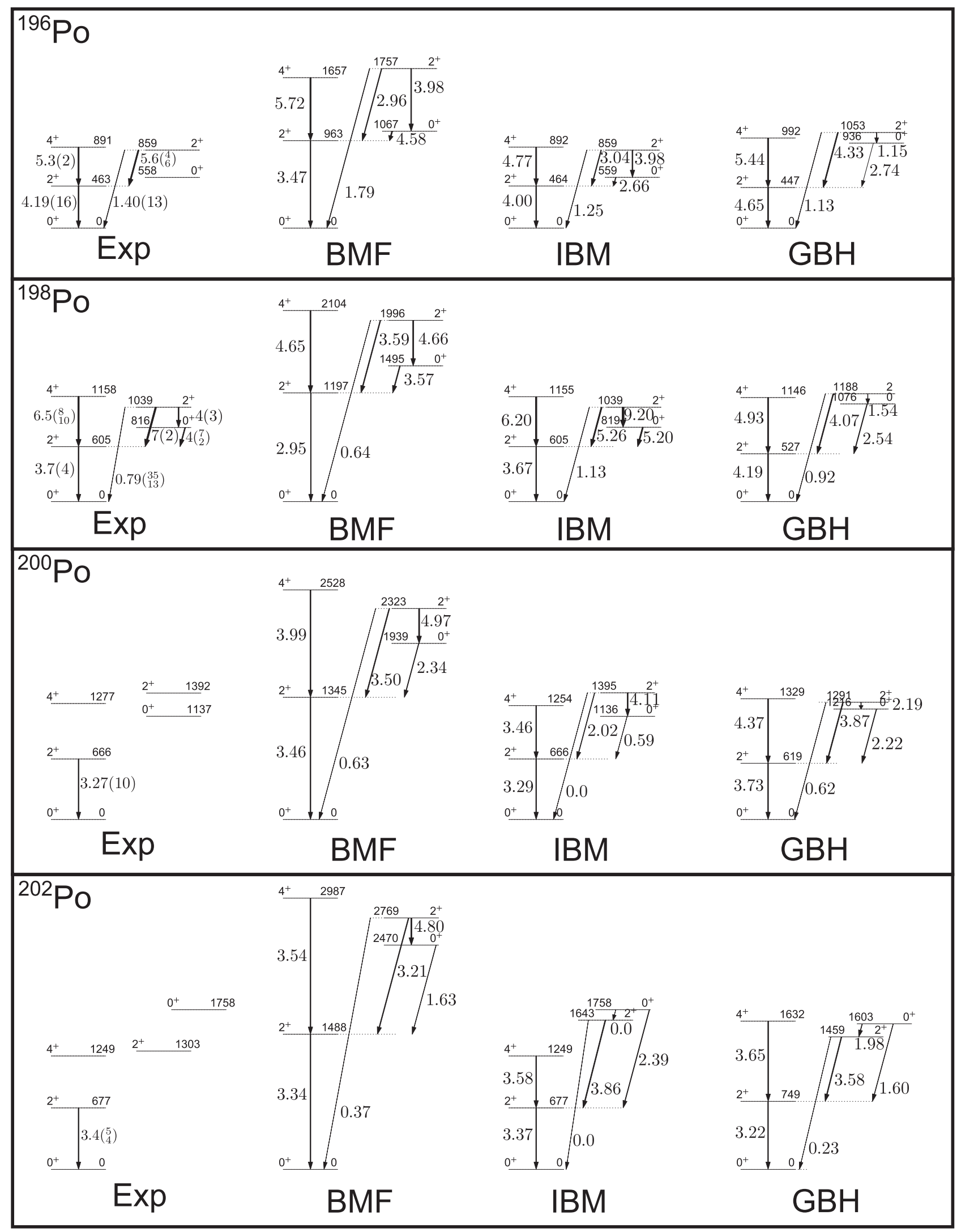

FIG. 15. Experimental levels of the low-lying structures in ${ }^{196,198,200,202}$ Po. Level energies (in keV) are taken from Nuclear Data Sheets. Transitional $\left|Q_{t}\right|$ values (in eb) are based on the experimentally determined matrix elements. The width of the arrows represents the relative size of the transitional quadrupole moments $\left|Q_{t}\right|$. Experimental level energies and $\left|Q_{t}\right|$ values are compared to the same information, predicted by the BMF [19], IBM [58], and GBH [59] models. 


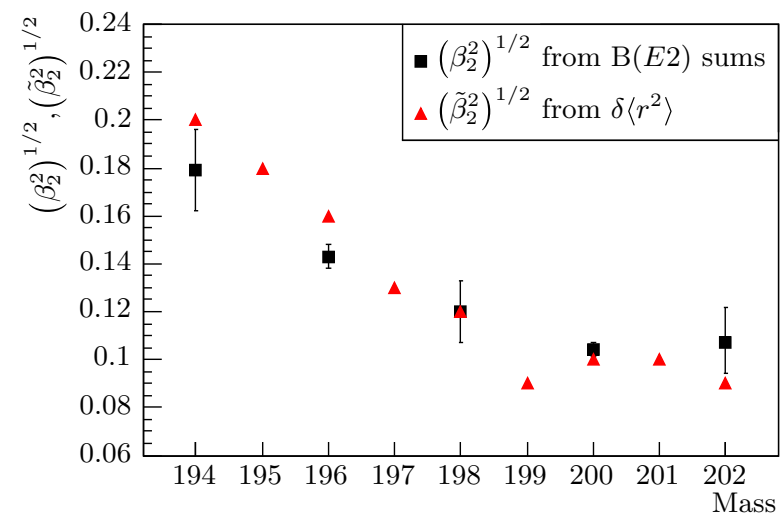

FIG. 16. (Color online) Deformation parameters of the ground state, extracted from the charge radii $\delta\left\langle r^{2}\right\rangle$ (triangles) [61] and sum of squared matrix elements according to Eq. (6) (squares). For odd isotopes the deformation parameter for the $3 / 2^{-}$ground state is shown. The data point for ${ }^{194} \mathrm{Po}$ is deduced from the lifetime measurement [15].

The experimentally determined transitional quadrupole moments $\left|Q_{t}\right|$ connecting the $2_{1}^{+}$and $2_{2}^{+}$states to the ground state are displayed and compared to the predictions from the BMF, IBM, and GBH calculations in Fig. 17. The same trend of increasing deformation with decreasing mass is observed from the experimental $\left|Q_{t}\right|$ values. The $\mathrm{BMF}\left|Q_{t}\left(2_{1}^{+} \rightarrow 0_{1}^{+}\right)\right|$ values start to deviate from the experimental values at mass $A=198$ as noted earlier. The inclusion of the new data points deduced in this work shows that the three theoretical approaches reproduce the experimental values quite well.

The reproduction of the experimental $\left|Q_{t}\left(2_{1}^{+} \rightarrow 0_{1}^{+}\right)\right|$ values by the IBM follows directly from the fit performed to the measured $B(E 2)$ values to fix the parameters of the IBM Hamiltonian. Nevertheless, the experimental trend in $\left|Q_{t}\left(2_{2}^{+} \rightarrow 0_{1}^{+}\right)\right|$is predicted well by the IBM, as well

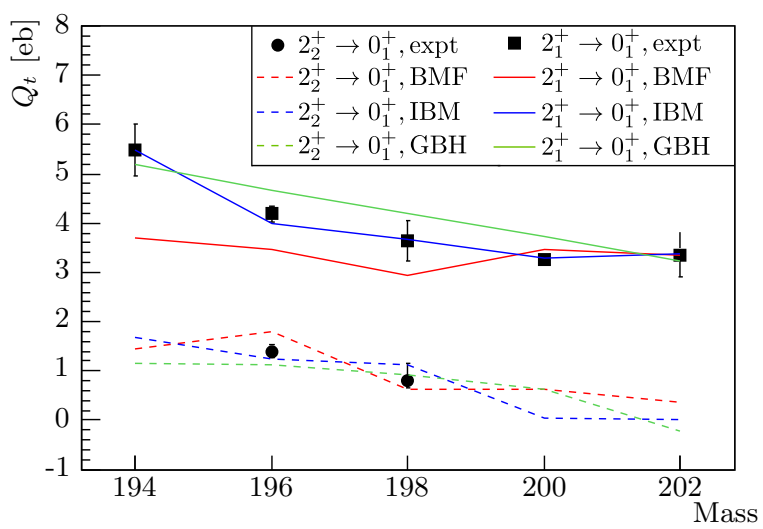

FIG. 17. (Color online) Experimental $\left|Q_{t}\right|$ values extracted from the measured matrix elements $\left\langle 0_{1}^{+}\|E 2\| 2_{1}^{+}\right\rangle$and $\left\langle 0_{1}^{+}\|E 2\| 2_{2}^{+}\right\rangle$in the even-even polonium nuclei as a function of the mass number. Data for $A=194$ are taken from [15]. Experimental values are compared to predictions from three theoretical model descriptions: the beyondmean-field (BMF) model [19], the interacting boson model (IBM) [58], and the general Bohr Hamiltonian (GBH) model [59].

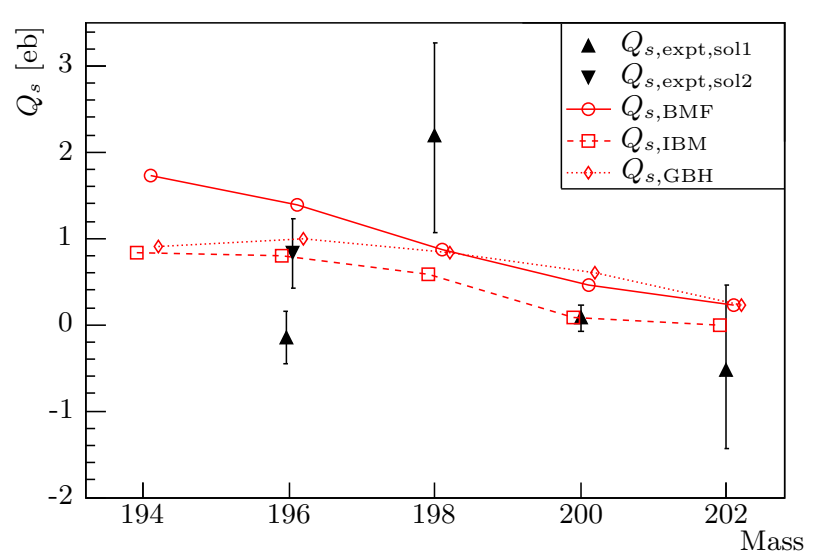

FIG. 18. (Color online) Experimentally determined values for the spectroscopic quadrupole moment $Q_{s}$ of the $2_{1}^{+}$state as a function of the mass of the polonium isotope. In ${ }^{196} \mathrm{Po}$ no model-independent distinction could be made between two solutions for the matrix elements, yielding two different results for $Q_{s}$. Both results are shown here with a small offset from integer $A$ for clarity. Experimental results are compared to predictions from the BMF model [19], IBM [58], and GBH model [59].

as the GBH and BMF models. The GBH model slightly overestimates the collectivity in the $2_{1}^{+} \rightarrow 0_{1}^{+}$transition for ${ }^{196-200}$ Po.

The deformation of the $2_{1}^{+}$state can be understood in a Coulomb-excitation experiment through the measurement of the spectroscopic quadrupole moment $Q_{s}$. The observed trend of increasing deformation in the $2_{1}^{+}$state when going down in mass number, shown in Fig. 18, is predicted by the three model descriptions.

A phenomenological two-state mixing model was used to calculate the $E 2$ matrix elements between low-lying states in the neutron-deficient ${ }^{182-188} \mathrm{Hg}$ isotopes $[3,26]$ to test the assumption that the excited states in the mercury isotopes can be described by a spin-independent interaction between two rotational structures. A common set of matrix elements within the unperturbed bands (transitional as well as diagonal E2 matrix elements) for the four studied mercury isotopes was found to reproduce most of the experimental results. A similar approach has been used for the polonium isotopes studied in this work. However, in the polonium isotopes a rotational structure was assumed to mix with a more spherical structure. A fit with the variable moment of inertia model [63] of the yrast $4^{+}, 6^{+}, 8^{+}$, and $12^{+}$levels in ${ }^{196}$ Po was used to determine the unperturbed energies of the $0^{+}$and $2^{+}$rotational states. The $10^{+}$state was not included because of an ambiguity. In this procedure, no mixing was assumed for states with spin $I \geqslant 4$. Using the unperturbed $0^{+}$and $2^{+}$rotational energies from the variable moment of inertia fit, information on the size of the spin-independent mixing matrix element was extracted [33]. The mixing amplitudes, listed in Table X, were determined by combining the spin-independent mixing matrix element $V=200 \mathrm{keV}$ with the mixed experimental level energies.

The experimental E2 matrix elements can then be expressed in terms of pure intraband matrix elements and a set of mixing amplitudes. No interband transitions between 
TABLE X. Square of wave-function mixing amplitudes of the "normal" (vibrational) configuration, at spin $0^{+}\left(\alpha_{0^{+}}^{2}\right)$ and spin $2^{+}$ $\left(\alpha_{2^{+}}^{2}\right)$. Details on the method applied to extract these values are provided in [33].

\begin{tabular}{lll}
\hline \hline Isotope & $\alpha_{0^{+}}^{2}$ & $\alpha_{2^{+}}^{2}$ \\
\hline${ }^{194} \mathrm{Po}$ & $12 \%$ & $29 \%$ \\
${ }^{196} \mathrm{Po}$ & $85 \%$ & $50 \%$ \\
${ }^{198} \mathrm{Po}$ & $94 \%$ & $69 \%$ \\
${ }^{200} \mathrm{Po}$ & $97 \%$ & $92 \%$ \\
${ }^{202} \mathrm{Po}$ & $99 \%$ & $88 \%$ \\
\hline \hline
\end{tabular}

the unperturbed structures were allowed. A set of unperturbed matrix elements was fitted to optimally reproduce the experimental results, yielding $\left\langle 0_{I}^{+}\|E 2\| 2_{I}^{+}\right\rangle=$ $1.1 \mathrm{eb},\left\langle 0_{I I}^{+}|| E 2|| 2_{I I}^{+}\right\rangle=1.5 \mathrm{eb},\left\langle 2_{I}^{+}\|E 2\| 2_{I}^{+}\right\rangle=-0.4 \mathrm{eb}$, and $\left\langle 2_{I I}^{+}\|E 2\| 2_{I I}^{+}\right\rangle=1.8 \mathrm{eb}$. Here, $I$ represents the spherical structure, and $I I$ the deformed one. In the fitting procedure the unperturbed diagonal matrix elements were not allowed to cross the rotational limit compared to the intraband transitional matrix element $\left(\left|\left\langle 2^{+}|| E 2|| 2^{+}\right\rangle\right|<1.19 \times\left\langle 0^{+} \| E 2|| 2^{+}\right\rangle[64]\right)$.

A comparison of the measured and calculated values of the E2 matrix elements is shown in Fig. 19. The best fit was found with solution 2 in ${ }^{196}$ Po (see Table IX), where the diagonal matrix element is positive and $\left\langle 0_{1}^{+}\|E 2\| 2_{2}^{+}\right\rangle$is negative. Most of the experimental results are reproduced within the $1 \sigma$ uncertainty. The total $\chi^{2}$ for this fit is equal to 102 , while the total $\chi^{2}$ for the best fit to solution 1 is equal to 189 . The extracted unperturbed $E 2$ matrix elements describing the rotational structure in the polonium isotopes are comparable

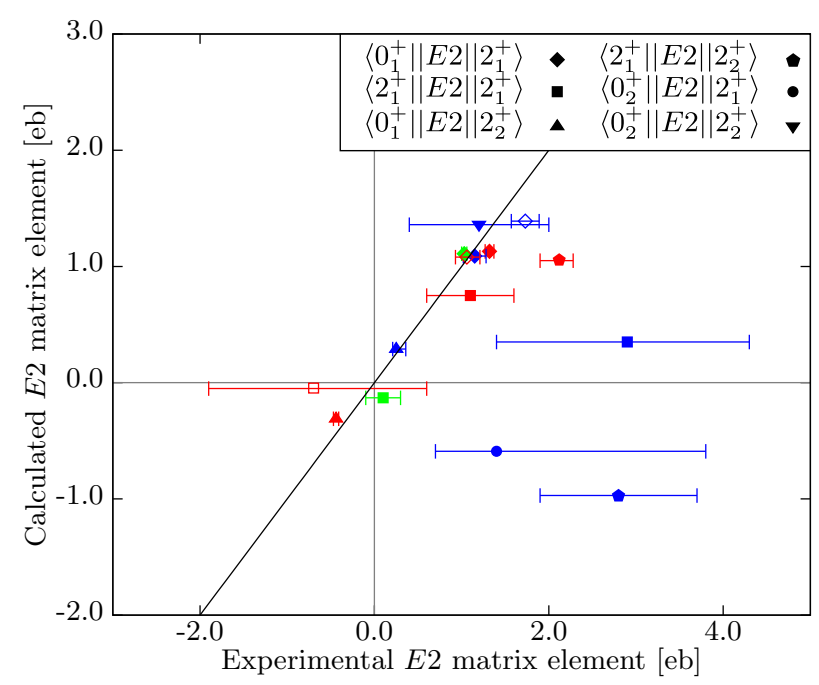

FIG. 19. (Color online) Measured E2 matrix elements determined in this work, compared to those extracted from two-level mixing calculations for ${ }^{196} \mathrm{Po}$ (filled red symbols), ${ }^{198} \mathrm{Po}$ (filled blue symbols), ${ }^{200} \mathrm{Po}$ (filled green symbol), and ${ }^{202} \mathrm{Po}$ (open red symbol). Measured $1 \sigma$ error bars are shown. In ${ }^{196}$ Po solution 2 (see Table IX) is adopted. The experimental E2 matrix element $\left\langle 0_{1}^{+}\|E 2\| 2_{1}^{+}\right\rangle$for ${ }^{194} \mathrm{Po}$ (open blue symbol) is deduced from the lifetime measurement [15]. to those extracted in the two-state mixing approach in the mercury isotopes for the weakly deformed oblate structure, where the extracted unperturbed transitional and diagonal $E 2$ matrix elements of the weakly deformed structure are 1.2 and $1.8 \mathrm{eb}$, respectively [3]. This supports the interpretation that a weakly deformed, oblate structure is intruding in the low-lying energy levels of the neutron-deficient polonium isotopes. The characteristics of this weakly deformed oblate structure seem to be related to those of the oblate structure in the mercury isotopes, which mirror the polonium isotopes with respect to $Z=82$.

\section{SUMMARY AND CONCLUSIONS}

A set of matrix elements coupling the low-lying states in the even-even neutron-deficient ${ }^{196-202}$ Po isotopes was extracted in two Coulomb-excitation campaigns, which were performed at the REX-ISOLDE facility at CERN. In the two heaviest isotopes, ${ }^{200,202} \mathrm{Po}$, the transitional and diagonal matrix elements of the $2_{1}^{+}$state were determined. In ${ }^{196,198} \mathrm{Po}$ multistep Coulomb excitation was observed to populate the $4_{1}^{+}, 0_{2}^{+}$, and $2_{2}^{+}$states. The relatively large uncertainty of the matrix elements related to the $0_{2}^{+}$and $2_{2}^{+}$states is due to the indirect observation of the $E 0$ transitions between the $0_{2}^{+}$and $0_{1}^{+}$states and the $2_{2}^{+}$and $2_{1}^{+}$states through characteristic polonium $\mathrm{x}$ rays. For future experiments the electron spectrometer SPEDE will provide a direct way of detecting $E 0$ transitions [65].

The experimental results were compared to the results from the measurement of mean-square charge radii in the polonium isotopes, confirming the onset of deformation from ${ }^{196} \mathrm{Po}$ onwards. Three model descriptions were used to compare to the data. Calculations with the BMF model, the IBM, and the GBH model show partial agreement with the experimental data. The comparison between the BMF model and the GBH results does not permit a firm conclusion regarding the effect of triaxial quadrupole deformations. Finally, calculations with a phenomenological two-level mixing model hint at the spin-independent mixing of a more spherical structure with a weakly deformed oblate structure. Overall the comparison to theory would benefit from an increase in the experimental sensitivity. This increased sensitivity could be reached in Coulomb-excitation experiments with higher beam energies at HIE-ISOLDE [66,67].

\section{ACKNOWLEDGMENTS}

We acknowledge the support of the ISOLDE Collaboration and technical teams and, especially, the support of RILIS and REX. This work was supported by FWO-Vlaanderen (Belgium), by GOA/2010/010 (BOF KU Leuven), by the Interuniversity Attraction Poles Programme initiated by the Belgian Science Policy Office (BriX network P7/12), by the European Commission within the Seventh Framework Programme through I3-ENSAR (Contract No. RII3-CT2010-262010), by the German BMBF under Contract Nos. 05P12PKFNE, 06DA9036I 05P12RDCIA, and 05P12RDCIB, by the UK Science and Technology Facilities Council, by the 
Spanish MINECO under Project No. FIS2011-28738-C02-02, by Narodowe Centrum Nauki (Polish Center for Scientific Research) Grant No. UMO-2013/10/M/ST2/00427, by the
Academy of Finland (Contract No. 131665), and by the European Commission through the Marie Curie Actions call PIEFGA-2008-219174 (J.P.).
[1] K. Heyde and J. L. Wood, Rev. Mod. Phys. 83, 1467 (2011).

[2] G. Ulm et al., Z. Phys. A 325, 247 (1986).

[3] N. Bree et al., Phys. Rev. Lett. 112, 162701 (2014).

[4] J. E. García-Ramos and K. Heyde, Phys. Rev. C 89, 014306 (2014).

[5] A. N. Andreyev et al., Nature 405, 430 (2000).

[6] R. Julin, K. Helariutta, and M. Muikku, J. Phys. G: Nucl. Part. Phys. 27, R109 (2001).

[7] H. De Witte et al., Phys. Rev. Lett. 98, 112502 (2007).

[8] M. Seliverstov et al., Phys. Lett. B 719, 362 (2013).

[9] A. Oros et al., Nucl. Phys. A 645, 107 (1999).

[10] N. Bijnens et al., Phys. Rev. Lett. 75, 4571 (1995).

[11] T. E. Cocolios et al., Phys. Rev. Lett. 106, 052503 (2011).

[12] W. Borchers et al., Hyperfine Interact. 34, 25 (1987).

[13] T. Hilberath et al., Z. Phys. A 342, 1 (1992).

[14] F. LeBlanc, D. Lunney, J. Obert, J. Oms, J. C. Putaux, B. Roussière, J. Sauvage, S. Zemlyanoi, J. Pinard, L. Cabaret, H. T. Duong, G. Huber, M. Krieg, V. Sebastian, J. E. Crawford, J. K. P. Lee, M. Girod, S. Péru, J. Genevey, and J. Lettry (ISOLDE Collaboration), Phys. Rev. C 60, 054310 (1999).

[15] T. Grahn et al., Phys. Rev. Lett. 97, 062501 (2006).

[16] T. Grahn et al., Phys. Rev. C 80, 014323 (2009).

[17] C. Ellegaard et al., Nucl. Phys. A 206, 83 (1973).

[18] L. Bernstein et al., Phys. Rev. C 52, 621 (1995).

[19] J. M. Yao, M. Bender, and P.-H. Heenen, Phys. Rev. C 87, 034322 (2013).

[20] J. E. García-Ramos, V. Hellemans, and K. Heyde, Phys. Rev. C 84, 014331 (2011).

[21] K. Helariutta et al., Eur. Phys. J. A 6, 289 (1999).

[22] K. Van de Vel et al., Eur. Phys. J. A 17, 167 (2003).

[23] G. D. Dracoulis, Phys. Rev. C 49, 3324 (1994).

[24] G. Lane et al., Nucl. Phys. A 589, 129 (1995).

[25] J. C. Walpe et al., Phys. Rev. C 85, 057302 (2012).

[26] L. P. Gaffney et al., Phys. Rev. C 89, 024307 (2014).

[27] D. Cline, Annu. Rev. Nucl. Particle Sci. 36, 683 (1986).

[28] O. Kester et al., Nucl. Instrum. Methods Phys. Res. B 204, 20 (2003).

[29] T. E. Cocolios et al., Nucl. Instrum. Methods Phys. Res. B 266, 4403 (2008).

[30] B. Marsh et al., Nucl. Instrum. Methods Phys. Res. B 317, 550 (2013).

[31] A. Kramida, Y. Ralchenko, J. Raeder, and NIST ASD Team, NIST Atomic Spectra Database (ver. 5.1) (NIST, Gaithersburg, MD, 2013).

[32] J. Van de Walle et al., Phys. Rev. C 79, 014309 (2009).

[33] N. Kesteloot, Ph.D. thesis, KU Leuven (2015).

[34] N. Warr et al., Eur. Phys. J. A 49, 40 (2013).

[35] A. N. Ostrowski et al., Nucl. Instrum. Methods Phys. Res. A 480, 448 (2002).
[36] N. Bree et al., Nucl. Instrum. Methods Phys. Res. B 360, 97 (2015).

[37] T. Grahn et al., EPJ Web Conf. 63, 01009 (2013).

[38] T. Kibédi, T. Burrows, M. Trzhaskovskaya, P. Davidson, and C. Nestor, Nucl. Instrum. Methods Phys. Res. A 589, 202 (2008).

[39] R. B. Firestone, Table of Isotopes, 8th ed., Number 4573810 (John Wiley \& Sons, New York, 1996).

[40] M. Klintefjord, forthcoming (2015).

[41] R. Stegmann, forthcoming (2015).

[42] D. Abriola and A. A. Sonzogni, Nucl. Data Sheets 107, 2423 (2006)

[43] S. Basu, G. Mukherjee, and A. Sonzogni, Nucl. Data Sheets 111, 2555 (2010).

[44] M. Zielinska et al., arXiv:1506.04633.

[45] N. Bijnens et al., Phys. Rev. C 58, 754 (1998).

[46] O. Helene, Nucl. Instrum. Methods 212, 319 (1983).

[47] T. Czosnyka, D. Cline, and C. Y. Wu, Bull. Am. Phys. Soc. 28, 745 (1983)

[48] T. Czosnyka, D. Cline, and C. Y. Wu, Coulomb excitation data analysis code GOSIA.

[49] S. Raman, C. Malarkey, W. Milner, C. Nestor, and P. Stelson, At. Data Nucl. Data Tables 36, 1 (1987).

[50] M. Luontama et al., Z. Phys. A 324, 317 (1986).

[51] C. Fahlander, L. Hasselgren, and J. Thun, Nucl. Instrum. Methods 146, 329 (1977).

[52] S. Raman, C. Nestor, and P. Tikkanen, At. Data Nucl. Data Tables 78, 1 (2001).

[53] P. Paradis, G. Lamoureux, R. Lecomte, and S. Monaro, Phys. Rev. C 14, 835 (1976).

[54] J. Barrette et al., Phys. Rev. C 6, 1339 (1972).

[55] K. Wrzosek-Lipska et al., Phys. Rev. C (2015) (unpublished).

[56] M. Lach et al., Z. Phys. A 350, 207 (1994).

[57] J. Wauters et al., Z. Phys. A 344, 29 (1992).

[58] J. E. García-Ramos and K. Heyde, Phys. Rev. C 92, 034309 (2015).

[59] L. Próchniak (private communication).

[60] L. Próchniak and S. G. Rohoziński, J. Phys. G: Nucl. Part. Phys. 36, 123101 (2009).

[61] M. D. Seliverstov et al., Phys. Rev. C 89, 034323 (2014).

[62] K. Wrzosek-Lipska et al., Phys. Rev. C 86, 064305 (2012).

[63] M. Mariscotti, G. Scharff-Goldhaber, and B. Buck, Phys. Rev. 178, 1864 (1969).

[64] A. Bohr, Rev. Mod. Phys. 48, 365 (1976).

[65] J. Konki et al., EPJ Web Conf. 63, 01019 (2013).

[66] M. Lindroos, P. Butler, M. Huyse, and K. Riisager, Nucl. Instrum. Methods Phys. Res. B 266, 4687 (2008).

[67] D. Voulot et al., IPAP-2012 Conf. Proc. C1205201, 3853 (2012). 